\title{
Annotated host catalogue for the Tachinidae (Diptera) of the Czech Republic
}

\author{
Jaromír Vaňhara*, Hans-Peter Tschorsnig, Benno Herting †े, Petr Mückstein \\ \& Veronika Michalková
}

Vaňhara, J., Tschorsnig, H.-P., Herting, B., Mückstein, P. \& Michalková, V. 2009: Annotated host catalogue for the Tachinidae (Diptera) of the Czech Republic. — Entomol. Fennica 20: 22-48.

An annotated host catalogue is given for the Tachinidae of the Czech Republic. It comprises 149 of 476 tachinid species which are currently known from this country (included the two new records cited below). 195 hosts are listed. The first host records of Tachinidae date back to the second half of the 19th century. The bibliography for the host records consists of 116 papers of 55 researchers. Several records of hitherto unpublished material are included. Phryxe setifacies and Anthomyiopsis plagioderae are first records for the Czech Republic.

J. Vaňhara (*corresponding author), Masaryk University, Faculty of Science, Kotlářská 2, CZ-61137 Brno, Czech Republic, vanhara@sci.muni.cz

H.-P. Tschorsnig, Staatliches Museum für Naturkunde, Rosenstein 1, D-70 191

Stuttgart, Germany, tschorsnig.smns@naturkundemuseum-bw.de

P. Mückstein Administration of the Protected Landscape Area Žd'árské vrchy, Brněnská 39, CZ-591 01 Žd'ár nad Sázavou, Czech Republic, muckstein @email.cz

V. Michalková, Masaryk University, Faculty of Science, Kotlárská 2, CZ-611 37

Brno, Czech Republic, vmichalkova@yahoo.com

Received 22 August 2007, accepted 21 January 2008

\section{Introduction}

Tachinidae are a very large and important dipteran family of (mainly) insect parasitoids. This study provides for the first time a critical host catalogue for Tachinidae of the Czech Republic. It is based on a compilation of literature records from the Czech Republic as well as on revisions of material (see below). There was a thorough search of literature, but there is nevertheless no guarantee that every record was found by the authors, because host records of Czech Tachinidae are very scattered in many journals, sometimes only appearing as marginal footnotes.
The tachinid species are listed in their actual valid nomenclature; probable misidentifications are - if possible - tentatively corrected, but the original name is always given in parenthesis, and a comment is added in case of corrections. Wrong or most probably wrong records that cannot be corrected are set in square brackets. Possible reasons for wrong records - which are not rare in literature - are either misidentifications of the tachinid or misassociated hosts or both. Misidentifications of tachinids were frequent, especially during the 19th and early 20th century, because of the lack of true specialists of this difficult group, but they still happen today when workers in ap- 
plied entomology hesitate to contact specialists. Wrong host associations easily happen in soil samples (forest litter) or in mass rearings when various hosts can be hidden among the plant material. Uncertainties in the tachinid identification can be solved when the tachinid material is still available for study, but even if a revision yields the correct identification of a tachinid, the host might nevertheless be wrong. Such cases cannot be solved, but they can be at least commented on or tentatively corrected in the light of the presentday knowledge of the host-range of the tachinid species (mainly based on revisions and compilations of European host records done by Hans-Peter Tschorsnig and the late Benno Herting).

References of the general parts (Introduction etc.) quoted below are cited in full. They can be found in the References of this paper, or if they include factual host information, they are listed in the section 5. "Host record bibliography".

\subsection{Short outline of the history of host/tachinid investigations in the Czech Republic}

Tachinids from the present-day Czech Republic have been studied since the first faunistic record from 1791 (Preyssler et al. 1793), but the knowledge of the diversity and especially of the hosts of this family is as incomplete as in most other European countries. The most recent information on Czech Tachinidae is given by Vaňhara et al. (2004), Tschorsnig et al. (2005), Vaňhara \& Tschorsnig (2006), and Mückstein et al. (2007).

The first data on tachinid hosts originate from the second half of the 19th century when the area of the present-day Czech Republic was part of the old Austrian-Hungarian Empire (Kirchner 1861; Wachtl 1882, 1886; Gold 1893, 1895; Mik \& Wachtl 1895). The rearings were first and foremost intiated by studies on parasitoids of insect pests which caused severe damage in coniferous forests during that time. Wachtl reared also many other lepidopterous hosts, but he published only a small part of it.

Brauer and Bergenstamm, both experts on Diptera, worked in the Museum at Vienna on Tachinidae at the end of the 19th century. They (Brauer \& Bergenstamm 1891, 1894) also pub- lished breeding records of Tachinidae, which they periodically received from other colleagues (e.g. R. von Stein). The material of the lepidopterist Richard Ritter von Stein (abbreviated "Rtt. v. Stein" or "R. v. Stein" in Brauer \& Bergenstamm 1891, 1894) most probably originates from W Bohemia, namely Chodov (=Chodau), nr. Karlovy Vary, where von Stein lived (Koleška 1993), but possibly also from other places in Bohemia where he collected (e.g. Sumava Mts. etc.).

The Czech entomologist A. Vimmer was the only one who worked on Tachinidae of lepidopterous hosts at the beginning of the 20th century, especially soon after Czechoslovakia was established (see Vimmer 1906-1938).

Tachinids were intensively studied by forestry researchers for pest control between World Wars I and II (Kolubajiv, Komárek, Pfeffer, Růžička). One result of this period was the first parasitoid catalogue on Lymantria monacha of Kolubajiv \& Pfeffer (1931) summarized also by Kolubajiv (1937), however with no exact information on localities. These older findings were later also used by Kudler (1954). Kolubajiv (1962) used them again, but he added additional breeding records.

At the end of the 1930s, some research was organized by foreigners to study parasitoids of European forest pests that had been introduced into Canada. Two papers on hosts in their pupal stage, which were massively exported from central European countries for purposes of biological control, were published later (Finlayson \& Finlayson 1958a, 1958b). The agriculture research branch, especially of the Czech sugar industry, searched also for new ways to combat sugar-beet pests using biological control methods (Rambousek 1928, 1929). Between 1933 and 1942, D. Jacentkovský recorded several hosts of tachinids in Moravia, though his papers were rather focused on faunistics with a special emphasis on practical forestry.

From the 1950s on, J. Čepelák systematically studied hosts, particularly in cooperation with $\mathrm{M}$. Čapek, and some others. Although their papers were predominantly focused on Slovakia, there were also some records from the Czech Republic. Applied forestry research also brought new knowledge about the hosts of tachinids during this period (Hochmut, Kalandra, Martinek, Ur- 
ban, etc.). Many records are scattered in various journals (Kletečka, Řezáč, Samšiňáková, Šámal, Weiser, Zuska, etc.).

Recently there have been attempts to systematically search for new hosts of tachinids (e.g. Mückstein et al. 2004, 2007). Our future projects will use molecular-genetic methods, which could perhaps help to explain phylogeny and possible coevolutionary interactions between Tachinidae and their hosts.

\subsection{Revisionary work}

Most revisionary work concerning reared Czech Tachinidae was done by the late B. Herting, who studied the old tachinid collections in NHMW during several visits in the late 1950 s. The results were integrated in his book on the "Biologie der westpaläarktischen Tachinidae" from 1960. The reared material stored in NHMW was - as far as it could be found - studied again during a visit by H.-P. Tschorsnig in 1988, leading to a few corrections and additions to Herting's results. The tachinid collection of the Forestry and Game Management Research Institute of Strnady near Prague (Výzkumný ústav lesního hospodářství a myslivosti) was revised by B. Herting between 1995 and 1997, and his unpublished list could be used for the present publication. The large collection of Wachtl was completely revised by H.-P. Tschorsnig and B. Herting during 1996 and 2002 (see Tschorsnig \& Herting 2005). Hence, it is clear that B. Herting - although deceased in 2004 - must be a coauthor of the present paper. Recent material sent by several Czech dipterists (M. Barták, J. Vaňhara, P. Mückstein etc.) was revised or identified by H.-P. Tschorsnig during the last fifteen years, and material (partly of the collection Čepelák) was also revised or identified by J. Vaňhara.

\section{Explanation of the layout of the lists}

"Annotated parasitoid-host list" (Section 3)

- Left column: The arrangement of subfamilies, tribes and species of the tachinids mainly fol- lows Herting \& Dely-Draskovits (1993), but updated nomenclature to this relevant catalogue is listed below. Names used in the original papers are always cited in parentheses when they were different (synonyms, different combinations, emendations, misspellings or misidentifications), with an explanatory comment if necessary.

- Right column: Hosts are arranged for each tachinid in the systematic order of the "Hostparasitoid list". The host names which were used in the original papers are cited after "-as" when they were different, followed by an explanatory comment if necessary. Wrong or presumably wrong hosts are set in square brackets "[ ].

"Host-parasitoid list" (Section 4)

- Left column: Host orders are arranged as follows: Lepidoptera, Coleoptera, Hymenoptera, Dermaptera. The arrangement and nomenclature of Lepidoptera follows Laštůvka \& Liška (2005), the one for Coleoptera corresponds with Jelínek (1993), and the arrangement of the hymenopteran families, genera and species is alphabetical, their valid scientific names according to http://www. faunaeur.org.

- Right column: The tachinids are arranged in order of their importance for the host, the most important or specific parasitoids are mentioned first, doubtful or very uncommon hosts have an asterisk "*”, wrong or presumably wrong hosts are cited in square brackets "[ ]".

"Host record bibliography" (Section 5)

- Only original records are cited, but in the case of revisions or later confirmatory studies of the same material, these papers are also mentioned. Simple citations or replications in some subsequent listings are cited only when they are of special interest. Citations of several workers from Baer (1921) are omitted because Baer's records are not of Czech origin. The reference numbers of the "Host record bibliography" are used only in the "Annotated parasitoid-host list". 
Abbreviations

$\mathrm{CZ} \quad$ Czech Republic

HE Herting

ident. identification

misid. misidentified/misidentification

new rec. new record(s) of hitherto unpublished material

prb. probable/probably

rev. $\quad$ revised/revision (= redetermined by a specialist)

s.a. see also (= references based on the same material, but cited because of some interest)

TS Tschorsnig

VA Vaňhara

Acronyms of Depositories

NHMW Naturhistorisches Museum Wien, Austria

VULH Výzkumný ústav lesního hospodářství a myslivosti (Strnady)
Recent tachinid nomenclature [nomenclature of Herting and Dely-Draskovits (1993) in square brackets]:

- Actia resinellae Schrank [syn. nudibasis Stein] - see Andersen (1996)

- Allophorocera [syn. Erycilla] - see Tschorsnig \& Richter (1998)

- Cleonice keteli Ziegler - not yet in Herting \& Dely-Draskovits, see Ziegler (2000)

- Hubneria [Huebneria] - valid spelling according to ICZN Art. 33.3.

- Phenicellia [included in Thelaira] - see Tschorsnig \& Herting (1994)

- Phytomyptera [syn. Elfia] - see Andersen (1988)

- Pseudopachystylum goniaeoides [gonioides] - valid spelling according to ICZN Art. 33.3.

- Smidtia [syn. Timavia] - see Shima (1996)

\section{Annotated parasitoid-host list}

\section{Subfamily Exoristinae}

\section{Exoristini}

Exorista fasciata (Fallén, 1820)

(misid. as Eutachina larvarum-see

2; misid. as Exorista larvarum-18)

(misid. as Eutachina larvarum-

2; misid. as Exorista larvarum-18)

Exorista larvarum (Linnaeus, 1758) (as Eutachina-100; Tachina-105)

(as Tachina)

(misid. as Microtachina erucarum Rdi.)

(as Tachina)

(as Eutachina-2)

(as Tachina)

(as Tachina)

(as Tachina)

(as Tachina; possibly misid. = Voria ruralis)

(as Eutachina-2)

(as Tachina)

(as Tachina)

(as Tachina)
-Ennomos erosarius Denis \& Schiff. (Lep. Geometridae) [6]

-Catocala fraxini Linnaeus (Lep. Noctuidae) [2-s.a. 18-rev. TS]

-Calliteara fascelina Linnaeus (Lep. Lymantriidae) [2-s.a. 18-as Dasychira-rev.TS; according to a note of $\mathrm{HE}$ labelled "Ch" =Chodov, i.e. material of R. v. Stein]

-Zygaena ephialtes Linnaeus (Lep. Zygaenidae) [100-s.a. 105]

-Zygaena punctum Ochsenheimer (Lep. Zygaenidae) [22]

-Zygaena sp. (Lep. Zygaenidae) [2-rev. TS]

-Malacosoma neustria Linnaeus (Lep. Lasiocampidae) [8]

-Euthrix potatoria Linnaeus (Lep. Lasiocampidae) [63]

-Cosmotriche lobulina Denis \& Schiff. (Lep. Lasiocampidae) [87]

-Macroglossum stellatarum Linnaeus (Lep. Sphingidae) [105-as Macroglossa]

-Nymphalis antiopa Linnaeus (Lep. Nymphalidae) [2-as Vanessa-rev. 18; cited as

"R. v. Stein" in 18, but this is neither indicated on the label nor in 2]

-Argynnis paphia Linnaeus (Lep. Nymphalidae) [105]

-Simyra albovenosa Goeze (Lep. Noctuidae) [106-as Arsilonche]

-Catocala fraxini Linnaeus (Lep. Noctuidae) [105]

-Autographa gamma Linnaeus (Lep. Noctuidae) [69 as Plusia]

-Autographa jota Linnaeus (Lep. Noctuidae) [2-as Plusia-rev. 18]

-Xylena exsoleta Linnaeus (Lep. Noctuidae) [68-as Calocampa exoleta]

-Hadula trifolii Hufnagel (Lep. Noctuidae) [68-as Mamestra trifolii Rott.]

-Ceramica pisi Linnaeus (Lep. Noctuidae) [63-as Melanchra]

-Agrotis segetum Denis \& Schiff. (Lep. Noctuidae) [68]

-Euproctis chrysorrhoea Linnaeus (Lep. Lymantriidae) [10-as phaeorrhoea, 39] 
(as Eutachina-2)

(as Tachina)

(as Eutachina vidua-100; Tachina vidua-105)

(as Tachina)

(as Eutachina l. and E. vidua-100, both

with the same rearing data; Tachina l.and

T. vidua-105; possibly misid. E. fasciata) -Arctia festiva Hufnagel (Lep. Arctiidae) [100-as hebe-s.a. 105]

Exorista grandis (Zetterstedt, 1844) (as

Parasetigena segregata; misid. $=$ prb.

E. grandis)

(as Tricholyga sorbillans)

(as sorbillans Wied.)

(as Tricholyga sorbillans Wied. =prb.

E. grandis or misid.)

Exorista deligata Pandellé, 1896 (as

Tricholyga abberans Strb.; prb. misid., species not known from $\mathrm{CZ}$ )

Exorista mimula (Meigen, 1824) (as

Chaetotachina nigricans-100; =misid., identity unknown)

(as Microtachina erucarum Rond.; prb. misid.)

(as Microtachina erucarum Rdi.-2; erucarum Rond.-18)

(misid. as Chaetotachina rustica-2 and

Exorista rustica-18)

Exorista rustica (Fallén, 1810) (as Tachina rustica $\mathrm{Mg}$. var.nigricans; prb. misid.)

(as Tachina-16, 75; prb. misid. =prb. E. larvarum)

(as Chaetotachina-2; ident. not

confirmed)

(as Chaetotachina-2)

(as Chaetotachina-2)

(as Chaetotachina-2)

Chetogena media Rondani, 1859 (as

Chaetogena; confirmed by TS)

Diplostichus janitrix (Hartig, 1838)

Stilpnotia ]

-Arctia caja Linnaeus (Lep. Arctiidae) $[68,105]$

-Saturnia pavonia Linnaeus (Lep. Saturniidae) [101-as carpini]

-Hyles euphorbiae Linnaeus (Lep. Sphingidae) [39-as Celerio]

-Deilephila elpenor Linnaeus (Lep. Sphingidae) [6]

-Lymantria monacha Linnaeus (Lep. Lymantriidae) [37]

-[Saturnia pavonia Linnaeus (Lep. Saturniidae)] [112]

-[Leucoma salicis Linnaeus (Lep. Lymantriidae)] [100]

-[Diprion pini Linnaeus (Hym. Diprionidae)] [39] (but "flavicomus" on label)-rev. TS] tachinid wrong]

-[Lymantria monacha Linnaeus (Lep. Lymantriidae)] [16, 75]

-Calophasia sp. (Lep. Noctuidae) [5]
Parasetigena silvestris (Robineau-

Desvoidy, 1863) (misid. as Masicera silvatica-16-see figures, 17; Parasetigena segregata or misid. Phorocera assimilis-37; Tachinidae sp.-44- identified in 45; segregata-27, 40, 45, 46, 47, 73, 74, $75,76,86,104,105)$
-Leucoma salicis Linnaeus (Lep. Lymantriidae) [2-rev 18-as Stilpnotia 4-as

-Lymantria monacha Linnaeus (Lep. Lymantriidae) [37-rev. HE, 73-s.a. 74, 105]

-Phragmatobia fuliginosa Linnaeus (Lep. Arctiidae) [100-as Spilosoma-s.a. 105]

-Cladius pectinicornis Geoffroy (Hym. Tenthredinidae) [2-as comari-rev. 18]

-Pristiphora pallidiventris Fallén (Hym. Tenthredinidae) [2-as Nematus flavicornis

-[Nymphalis polychloros Linnaeus (Lep. Nymphalidae)] [105-as Vanessa] -[Cosmia trapezina Linnaeus (Lep. Noctuidae)] [39-as Calymnia; either host or

-Rhogogaster viridis Linnaeus (Hym. Tenthredinidae) [2-as Tenthredo scalaris]

-Tenthredo arcuata Forster (Hym. Tenthredinidae) [2-as Allantus notus-rev. 18]

-Tenthredopsis coquebertii Klug (Hym. Tenthredinidae) [2-rev. 18]

-Tenthredopsis scutellaris Fabricius (Hym. Tenthredinidae) [2-rev. 18-as palmata]

-Diprion pini Linnaeus (Hym. Diprionidae) [2-as Lophyrus-rev. 18-as Diprion 14, 42, 87]

-Diprion similis Hartig (Hym. Diprionidae) [2-as Lophyrus-rev. 18]

-Gilpinia frutetorum Fabricius (Hym. Diprionidae) [2-as Lophyrus-rev. 18-as

Diprion]

-Gilpinia hercyniae Hartig (Hym. Diprionidae) [2-as Lophyrus]

-Gilpinia polytoma Hartig (Hym. Diprionidae) [15-as Diprion polytomum]

-Gilpinia variegata Hartig (Hym. Diprionidae) [2-as Lophyrus variegatus-rev. 18-as Diprion]

-Gilpinia virens Klug (Hym. Diprionidae) [2-as Lophyrus-rev. 18-as Diprion]

-Lymantria monacha Linnaeus (Lep. Lymantriidae) [16, 17, 27-s.a. 30, 32-as

Liparis, 37-rev. HE 40, 44, 45, 46, 47, 73, 74, 75, 76, 86, 104-s.a. 105, new rec. - 
Phorocera assimilis (Fallén, 1810)

(as Setigera -100 ; prb. misid., identity unknown)

Phorocera obscura (Fallén, 1810) (as caesifrons)

(as Chaetogena vernalis-30, 32)

(as Chaetogena vernalis)

(as caesifrons)

(as Setigera caesifrons-100; caesifrons105 ; prb. misid., identity unknown)

Bessa parallela (Meigen, 1824)

(as selecta; prb. misid. $=$ prb. parallela $)$

(misid. as selecta-39)

(as Ptychomyia)

Bessa selecta (Meigen, 1824)

(as Ptychomyia)

(misid. as Prosopodes fugax)

(as Ptychomyia)

\section{Blondeliini}

Belida angelicae (Meigen, 1824) (as Ceromasia-26; Aporotachina-30)

Meigenia incana (Fallén, 1810) (prb. misid. =prb. Meigenia uncinata) Meigenia mutabilis (Fallén, 1810) (as bisignata Meig.; might be misid. because the typical parasitoid of Agelastica alni is Meigenia uncinata) Zaira cinerea (Fallén, 1810) (as Viviana) Medina collaris (Fallén, 1820)

Medina luctuosa (Meigen, 1824) (prb. misid.)

(prb. misid.)

(prb. misid.; erroneously given as Meigenia mutabilis in the English summary)

Medina melania (Meigen, 1824) (prb. misid.)

Staurochaeta albocingulata (Fallén, 1820) (as gracilis Egg.-2)

Leiophora innoxia (Meigen, 1824)

(as Hypostena-79; Arrhinomyia-11)
CZ, Doksy Krupá + Halamky, leg. Kolubajiv?, 1948, VULH-rev. HE] -Malacosoma neustria Linnaeus (Lep. Lasiocampidae) [105] -[Aglais urticae Linnaeus (Lep. Nymphalidae)] [105-as Vanessa; questionable host] -[Lymantria monacha Linnaeus (Lep. Lymantriidae)] [105; either host wrong or tachinid misid. possibly Parasetigena silvestris]

-[Arctia festiva Hufnagel (Lep. Arctiidae)] [100 - as hebe-s.a. 105]

-[Malacosoma neustria Linnaeus (Lep. Lasiocampidae)] [105; questionable host] -Operophtera brumata Linnaeus (Lep. Geometridae) [30-s.a. 32-as Cheimatobia, 61] - Operophtera fagata Scharfenberg (Lep. Geometridae) [30-as Cheimatobia boreata] -[Lymantria monacha Linnaeus (Lep. Lymantriidae)] [74, 105; prb. either host or tachinid ident. wrong]

-[Arctia festiva Hufnagel (Lep. Arctiidae)] [100-as hebe-s.a. 105]

-Yponomeuta evonymella Linnaeus (Lep. Yponomeutidae) [87, new rec.-CZ, Žatec (=Saaz), NHMW-rev. TS]

-Yponomeuta padella Linnaeus (Lep. Yponomeutidae) [30-as Hyponemeuta] -Tortrix viridana Linnaeus (Lep. Tortricidae) [39-rev. HE, new rec.-CZ, Šárka, leg. Kolubajiv?, 1944, VULH-rev. HE]

-Acleris ferrugana Denis \& Schiff. (Lep. Tortricidae) [83-as Acalla ferrugana Tr.] -Gilpinia polytoma Hartig (Hym. Diprionidae) [15-as Diprion polytomum-rev. HE (1 sp.) in VULH]

-Cladius pallipes Serville (Hym. Tenthredinidae) [2-as Priophorus albipes-rev. 18as Priophorus padi]

-Hemichroa crocea Geoffroy (Hym. Tenthredinidae) [2-as rufa-rev. 18]

-Nematus melanaspis Hartig (Hym. Tenthredinidae) [88]

-Pristiphora erichsonii Hartig (Hym. Tenthredinidae) [80-as Nematus]

-Arge sp. (Hym. Argidae) [26-as Arge berberidis Schrk.-s.a. 30-as Arge rosea; the records have the same data, but the host is cited under different species names so the exact identity remains unclear]

-[Agelastica alni Linnaeus (Col. Chrysomelidae)] [92]

-Gastrophysa viridula De Geer (Col. Chrysomelidae) [63]

-Agelastica alni Linnaeus (Col. Chrysomelidae) [79]

-Zabrus tenebrioides Goeze (Col. Carabidae) [77-only larvae]

-Galerucella lineola Fabricius (Col. Chrysomelidae) [96]

-Lochmaea capreae Linnaeus (Col. Chrysomelidae) [new rec.-CZ, Polnička. 1996 and Bílovice n.Sv., 2006, leg. Urban, det. Čepelák, VA]

-[Plagiodera versicolora Laicharting (Col. Chrysomelidae)] [94; the true Medina luctuosa is a parasitoid of Altica spp., whereas Plagiodera is a typical host of Medina separata]

-[Linaeidea aenea Linnaeus (Col. Chrysomelidae)] [93]

-[Gonioctena quinquepunctata Fabricius (Col. Chrysomelidae)] [90; confirmed hosts of $M$. luctuosa are Altica spp.]

-[Plagiodera versicolora Laicharting (Col. Chrysomelidae)] [94; hosts of Medina melania are not yet confirmed, but Plagiodera is a typical host of Medina separata]

-Monoctenus juniperi Linnaeus (Hym. Diprionidae) [2-as Lophyrus-rev. 18]

-[Agelastica alni Linnaeus (Col. Chrysomelidae)] [79-s.a. 11; either host wrong or tachinid misid. because L. innoxia is a parasitoid of Tetrigidae (Orthoptera)] 
Admontia grandicornis (Zetterstedt, 1849) (misid. as Trichoparia seria Mg.)

Blondelia inclusa (Hartig, 1838)

(as Ceromasia-42)

(as Lophyromyia-39)

Blondelia nigripes (Fallén, 1810)

(as Lydella)

(as Ceromasia)

(as Lydella-37; according to an unpubl. note of HE misid. by Fahringer as Phorocera assimilis $-81,82$ )

(as Lydella)

(as Lydella-41)

(as Ceromasia)

(as Lydella)

Blondelia piniariae (Hartig, 1838) (as Lydella nigripes-37, 82-prb. misid. = prb. piniariae; Tachina-58)

Compsilura concinnata (Meigen, 1824) (as Chaetogena rufipalpis-105; s. note under Acronicta aceris)

(as Phorocera)

(as Phorocera rufipalpis Mg.-100; Chaetogena rufipalpis-105; s.a. note under Acronicta aceris) (as Machaira serriventris Rd.-101)

(as Machaira serriventris Rd.) (as Chaetogena rufipalpis; s. note under Acronicta aceris)

(as Machaira serriventris Rd.)

(as Phorocera rufipalpis Mg.-100; Chaetogena rufipalpis-105; =nomen dubium, but according to unpubl. note of HE prb. C. concinnata) (as Machaira serriventris $\mathrm{Rd}-2$ )
-[Epinotia tedella Clerck (Lep. Tortricidae)] [39-rev. HE; from soil samples-host must be wrong because $A$. grandicornis is a parasitoid of Tipulidae]

-Diprion pini Linnaeus (Hym. Diprionidae) [42]

-Gilpinia polytoma Hartig (Hym. Diprionidae) [15-as Diprion polytomum 39-rev. $\mathrm{HE}]$

-Neodiprion sertifer Geoffroy (Hym. Diprionidae) $[\mathbf{5 7}, \mathbf{6 7}, \mathbf{9 8}]$

-Yponomeuta evonymella Linnaeus (Lep. Yponomeutidae) [39-as Hyponomeuta, new rec.-CZ, leg. Kudler, 1956, VULH-rev. HE]

-Yponomeuta padella Linnaeus (Lep. Yponomeutidae) [105]

-[Paranthrene tabaniformis Rottemburg (Lep. Sesiidae)] [39; host must be wrong] -Cochylimorpha hilarana Herrich-Schäffer (Lep. Tortricidae) [110-as Conchylis]

-Tortrix viridana Linnaeus (Lep. Tortricidae) [39-rev. HE]

-Choristoneura murinana Hübner (Lep. Tortricidae) [37-as Cacoecia-rev. HE, 81s.a. $\mathbf{8 2}$ - as Cacoecia]

-Pandemis cerasana Hübner (Lep. Tortricidae) [39-as ribeana-rev. HE]

-Zeiraphera griseana Hübner (Lep. Tortricidae) [37-as Semasia diniana-rev. HE

65-as Enarmonia diniana-s.a. 66]

-Malacosoma neustria Linnaeus (Lep. Lasiocampidae) [8]

-Dendrolimus pini Linnaeus (Lep. Lasiocampidae) [41-s.a. 39]

-Inachis io Linnaeus (Lep. Nymphalidae) [28-as Vanessa]

-Aglais urticae Linnaeus (Lep. Nymphalidae) [25]

-Ennomos autumnarius Werneburg (Lep. Geometridae) [4]

-Operophtera brumata Linnaeus (Lep. Geometridae) [61]

-Xylena exsoleta Linnaeus (Lep. Noctuidae) [87]

-Ceramica pisi Linnaeus (Lep. Noctuidae) [105-as Mamestra]

-Arctia caja Linnaeus (Lep. Arctiidae) [4]

-Gilpinia polytoma Hartig (Hym. Diprionidae) [15-as Diprion polytomum]

-Bupalus piniarius Linnaeus (Lep. Geometridae) [37, 58-rev. 87, 82]

-Malacosoma neustria Linnaeus (Lep. Lasiocampidae) [39-rev. HE 105]

-Bombyx mori Linnaeus (Lep. Bombycidae) [13-as "Seidenspinner"]

-Sphinx ligustri Linnaeus (Lep. Sphingidae) [100-s.a. 105]

-Iphiclides podalirius Linnaeus (Lep. Papilionidae) [101-as Papilio-s.a. 104, 105]

-Pieris brassicae Linnaeus (Lep. Pieridae) [37-rev. HE 39-rev. HE 72, 105]

-Pieris rapae Linnaeus (Lep. Pieridae) [105]

-Pieris napi Linnaeus (Lep. Pieridae) [105]

-Nymphalis antiopa Linnaeus (Lep. Nymphalidae) [105-as Vanessa]

-Inachis io Linnaeus (Lep. Nymphalidae) [2-as Vanessa jo]

-Aglais urticae Linnaeus (Lep. Nymphalidae) [105-as Vanessa]

-Vanessa atalanta Linnaeus (Lep. Nymphalidae) [105-as Pyrameis]

-Araschnia levana Linnaeus (Lep. Nymphalidae) [2-as Vanessa-rev. 18; "R. v.

Stein" on label according to $\mathrm{HE}, 4]$

-Phalera bucephala Linnaeus (Lep. Notodontidae) [72]

-Acronicta psi Linnaeus (Lep. Noctuidae) [72]

-Acronicta aceris Linnaeus (Lep. Noctuidae) [100-as Acronycta-s.a. 105]

-Lacanobia oleracea Linnaeus (Lep. Noctuidae) [2-as Mamestra-rev. 18]

-Calliteara pudibunda Linnaeus (Lep. Lymantriidae) [87] 
(as Compsilura sp.-105)

(as Machaira serriventris Rd.-2)

(as Machaira serriventris $\mathrm{Rd}-2$ )

\section{Acemyini}

Acemya acuticornis (Meigen, 1824)

\section{Winthemiini}

Winthemia cruentata (Rondani, 1859) (as ligustri Stein)

(as ligustri Stein-6; ident. not confirmed, might also belong to W. rufiventris Macq.) -Mimas tiliae Linnaeus (Lep. Sphingidae) [6]

Winthemia quadripustulata (Fabricius, 1794)

(as Chaetolyga-2)

(as Chaetolyga analis Mcq.-2)

(as Chaetolyga erythrura-100; quadripustulata var. erythrura-105; prb. misid., $W$. erythrura not known from $\mathrm{CZ}$ ) (as Chaetolyga-2)

Winthemia rufiventris (Macquart, 1849) (as xanthogastra; prb. misid., species not known from $\mathrm{CZ}$ )

Nemorilla floralis (Fallén, 1810)

Nemorilla maculosa (Meigen, 1824) (prb. correct)

Smidtia amoena (Meigen, 1824) (as Winthemia)

\section{Eryciini}

Aplomya confinis (Fallén, 1820)

(as Exorista)

Phebellia clavellariae (Brauer \& Bergenstamm, 1891) (as Parexorista-1)

Phebellia glauca (Meigen, 1824) (misid. as Parexorista glirina Rdi.-2) Phebellia glirina (Rondani, 1859) (misid. as Parexorista grossa-2) Phebellia triseta (Pandellé, 1896) (prb. misid., identity unknown)

Nilea hortulana (Meigen, 1824) (misid. as Parexorista polychaeta Mcq.-2) (as Exorista; prb. misid. $=$ prb.
-Euproctis chrysorrhoea Linnaeus (Lep. Lymantriidae) [39-rev. HE] -Lymantria monacha Linnaeus (Lep. Lymantriidae) [37, 105] -Cimbex sp. (on Salix caprea) (Hym. Cimbicidae) [2-as "Cimbex axillaris"-rev. 18] -Trichiocampus grandis Serville (Hym. Tenthredinidae) [2-as eucera Klg.-rev. 18-as viminalis Fall.]

-[Tortrix viridana Linnaeus (Lep. Tortricidae)] [39; either tachinid or host wrong] -[Rhyacionia buoliana Denis \& Schiff. (Lep. Tortricidae)] [39-as Evetria; either tachinid or host wrong because $A$. acuticornis is a parasitoid of Orthoptera]

-Sphinx ligustri Linnaeus (Lep. Sphingidae) $[6,31,39]$

-Deilephila elpenor Linnaeus (Lep. Sphingidae) [105-as Chaerocampa]

-Zerynthia polyxena Denis \& Schiff. (Lep. Papilionidae) [105-as Thais]

-Inachis io Linnaeus (Lep. Nymphalidae) [39]

-Aglais urticae Linnaeus (Lep. Nymphalidae) [105-as Vanessa]

-Argynnis aglaja Linnaeus (Lep. Nymphalidae) [2-rev. 18, 105]

-Cucullia lactucae Denis \& Schiff. (Lep. Noctuidae) [63]

-Cucullia umbratica Linnaeus (Lep. Noctuidae) [2-rev. 18]

-Shargacucullia scrophulariae Denis \& Schiff. (Lep. Noctuidae) [105-as Cucullia]

-Shargacucullia verbasci Linnaeus (Lep. Noctuidae) [100-as Cucullia-s.a. 105]

-Ceramica pisi Linnaeus (Lep. Noctuidae) [2-as Mamestra-rev. 18]

-Cerapteryx graminis Linnaeus (Lep. Noctuidae) [84-as Charaeas]

-[Dioryctria abietella Denis \& Schiff. (Lep. Pyralidae)] [112; host impossible for a large tachinid as Winthemia]

-Tortrix viridana Linnaeus (Lep. Tortricidae) [39-rev. HE]

-Choristoneura murinana Hübner (Lep. Tortricidae) [37-rev. HE]

-Zeiraphera griseana Hübner (Lep. Tortricidae) [37-as Semasia diniana, 65-as

Enarmonia diniana-s.a. 66]

-Trachycera suavella Zincken (Lep. Pyralidae) [87]

-Panolis flammea Denis \& Schiff. (Lep. Noctuidae) [105-as griseovariegata Goeze]

-Aporia crataegi Linnaeus (Lep. Pieridae) [105-questionable host]

-Neozephyrus quercus Linnaeus (Lep. Lycaenidae) [4-as Zephyrus]

-Pseudoclavellaria amerinae Linnaeus (Hym. Cimbicidae) [1-rev. 19; the material ex "Cimbex ariabilis" is not from R.v.Stein, as it is erroneously indicated in 18 , corrected in 19]

-Acronicta tridens Denis \& Schiff. (Lep. Noctuidae) [2-as Acronycta-rev. 18]

-Abia sericea Linnaeus (Hym. Cimbicidae) [2-rev. 18]

-[Choristoneura murinana Hübner (Lep. Tortricidae)] [37-as Cacoecia]

-Griposia aprilina Linnaeus (Lep. Noctuidae) [6-as Dichonia]

-Acronicta tridens Denis \& Schiff. (Lep. Noctuidae) [2-as Acronycta-rev. 18] 
Myxexoristops sp.)

Buquetia musca Robineau-Desvoidy, 1847 (as Eupogona setifacies-39; Epicampocera setifacies-105)

Phryxe erythrostoma (Hartig, 1838) (as Tachina-35; misid. as vulgaris-39)

Phryxe magnicornis (Zetterstedt, 1838)

(misid. as vulgaris)

(misid. as vulgaris)

Phryxe nemea (Meigen, 1824)

(as Blepharidopsis)

Phryxe prima (Brauer \& Bergenstamm, 1889)

(as Ceratochaetops)

Phryxe semicaudata Herting, 1959

Phryxe setifacies (Villeneuve, 1910)

(misid. as Blepharidopsis nemea)

Phryxe vulgaris (Fallén, 1810) (possibly misid.)

(=vulgaris or misid.)

(as Blepharidea-2)

(as Exorista-100)

(as Exorista-100, Blepharidea-102)

(as Blepharidea-2)

(as Blepharidea-2)

(prb. misid. =prb. Hubneria affinis)

(prb. misid. =prb. Hubneria affinis)

Periarchiclops scutellaris (Fallén, 1820)

(as Prosopaea abbreviata-2)

Bactromyia aurulenta (Meigen, 1824)

Pseudoperichaeta nigrolineata (Walker,

1853) (misid. as Exorista mitis in part-39) -Tortrix viridana Linnaeus (Lep. Tortricidae) [39-rev. HE, new rec.-CZ, Moravia,

Tvrdonice, leg. F. Gregor, 1954, VULH-rev. HE]

(as insidiosa R.-D.-4, Exorista roseanae

B.\& B.-20)

(as P. major)

(as P. major)

-[Cephalcia abietis Linnaeus (Hym. Pamphiliidae)] [34]

-Papilio machaon Linnaeus (Lep. Papilionidae) $[39,105]$

-Sphinx pinastri Linnaeus (Lep. Sphingidae) [35, 39-as Hyloicus rev. HE, new rec.CZ, Český Rudolec, leg. Kolubajiv?, 1934 and Psovlky nr. Rakovník, leg. Martinek, VULH-rev. HE]

-Zygaena laeta Hübner (Lep. Zygaenidae) [23]

-Tortrix viridana Linnaeus (Lep. Tortricidae) [39-rev. HE]

-Erannis defoliaria Clerck (Lep. Geometridae) [37-as Hibernia-rev. HE]

-Choristoneura murinana Hübner (Lep. Tortricidae) [18-as Cacoecia 81-s.a. 82-rev.]

-Pieris brassicae Linnaeus (Lep. Pieridae) [18]

-Neozephyrus quercus Linnaeus (Lep. Lycaenidae) [4-as Zephyrus]

-Abraxas grossulariata Linnaeus (Lep. Geometridae) [2-rev. 18]

-Zygaena carniolica Scopoli (Lep. Zygaenidae) [63]

-Zygaena punctum Ochsenheimer (Lep. Zygaenidae) [22]

-Thaumetopoea processionea Linnaeus (Lep. Thaumetopoeidae) [87]

-Zygaena angelicae Ochsenheimer (Lep. Zygaenidae) [6-rev. VA; first record of Phryxe setifacies for $\mathrm{CZ}]$

-Yponomeuta padella Linnaeus (Lep. Yponomeutidae) [105]

-Malacosoma neustria Linnaeus (Lep. Lasiocampidae) [8]

-Lasiocampa trifolii Denis \& Schiff. (Lep. Lasiocampidae) [105-s.a. 108]

-Deilephila porcellus Linnaeus (Lep. Sphingidae) [105-as Metopsilus]

-Aporia crataegi Linnaeus (Lep. Pieridae) [39]

-Pieris brassicae Linnaeus (Lep. Pieridae) [2-rev. 18, 105]

-Pieris napi Linnaeus (Lep. Pieridae) [105]

-Anthocharis cardamines Linnaeus (Lep. Pieridae) [105-as Euchloë]

-Satyrium pruni Linnaeus (Lep. Lycaenidae) [4-as Thecla]

-Nymphalis polychloros Linnaeus (Lep. Nymphalidae) [37-as Vanessa-rev. HE]

-Nymphalis xanthomelas Denis \& Schiff. (Lep. Nymphalidae) [105-as Vanessa]

-Nymphalis antiopa Linnaeus (Lep. Nymphalidae) [18-as Vanessa]

-Inachis io Linnaeus (Lep. Nymphalidae) [100-as Vanessa-s.a. 105, 108]

-Aglais urticae Linnaeus (Lep. Nymphalidae) [4-as Vanessa, 100-as Vanessa-s.a.

102, 104, 105, 108; new rec.-CZ, Žatec (=Saaz), NHMW-rev. TS]

-Araschnia levana Linnaeus (Lep. Nymphalidae) [18]

-Issoria lathonia Linnaeus (Lep. Nymphalidae) [2-as Argynnis-rev. 18]

-[Bupalus piniarius Linnaeus (Lep. Geometridae)] [82; questionable host]

-Autographa gamma Linnaeus (Lep. Noctuidae) [87-as Plusia]

-Tholera cespitis Denis \& Schiff. (Lep. Noctuidae) [2-as Neuronia-rev. 18-as

Epineuronia]

-Agrotis segetum Denis \& Schiff. (Lep. Noctuidae) [70-s.a. 105, 108]

- Orgyia antiqua Linnaeus (Lep. Lymantriidae) [105]

-[Parasemia plantaginis Linnaeus (Lep. Arctiidae)] [105]

-[Arctia caja Linnaeus (Lep. Arctiidae)] [108]

-Conistra rubiginea Denis \& Schiff. (Lep. Noctuidae) [2-as Acronycta rubiginosarev. 18-as Orrhodia ]

-[Pristiphora abietina Christ (Hym. Tenthredinidae)] [39; either parasitoid or host must be wrong]

-Archips crataegana Hübner (Lep. Tortricidae) [4, 20-as Cacoecia]

-[Malacosoma neustria Linnaeus (Lep. Lasiocampidae)] [39; host prb. wrong]

-[Euproctis chrysorrhoea Linnaeus (Lep. Lymantriidae)] [39; host prb. wrong] 
Lydella stabulans (Meigen, 1824)

Lydella thompsoni Herting, 1959 Cadurciella tritaeniata (Rondani, 1859) (as Parexorista-2, as Chaetina-18) Drino bohemica Mesnil, 1949 (as Sturmia inconspicua-rev. HE, but ident. uncertain because of the bad shape of the material)

Drino gilva (Hartig, 1838) (as

Argyrophylax gilva Htg.-2)

Drino inconspicua (Meigen, 1830) (as Argyrophylax-39; Sturmia bimaculata Htg.-41) (as Argyrophylax-39; Sturmia bimaculata Hart.-73, 74)

(misid. as Hemimasicera vicina-39)

(as Argyrophylax bimaculata Htg.-2) (as Sturmia-59)

(as Argyrophylax bimaculata Htg.-2) (as Argyrophylax bimaculata Htg.-2) (as Argyrophylax bimaculata Htg.-2; Sturmia-37, 38)

Drino lota (Meigen, 1824) (misid. as Winthemia quadripustulata-39; misid. Ernestia radicum-106, the figures clearly indicate D. lota)

(as Phorcida)

Hubneria affinis (Fallén, 1810) (as Parexorista polycheta-100, Exorista polychaeta and E. affinis-105, Exorista108)

(as Exorista)

(as Exorista)

(as Exorista)

(as Exorista)

(as Exorista)

(as Parexorista polycheta and Exorista

affinis-100; Exorista affinis and $E$.

polycheta-105; Exorista-108)

(as Parexorista polycheta-100, 104;

Exorista affinis and E. polycheta-105;

Exorista-108)

(as Parexorista polycheta Macq. and

Exorista affinis -101; Exorista polycheta-

105; Exorista-108)

(as Exorista-100; Parexorista-102;

Parexorista polycheta-104; Exorista-

105, 108)

Carcelia atricosta Herting, 1961 (misid. as Sisyropa lucorum-2 and C.

puberula-18)

(misid.as Parexorista cheloniae-2 and

C. puberula-18)
-Hydraecia micacea Esper (Lep. Noctuidae) [18-specimen in NHMW labelled

"Saaz" (=Žatec), according to an unpublished note of HE 63]

-Ostrinia nubilalis Hübner (Lep. Pyralidae) [3]

-Callophrys rubi Linnaeus (Lep. Lycaenidae) [2-as Thecla-rev. 18]

-Gilpinia hercyniae Hartig (Hym. Diprionidae) [37-as Lophyrus hercyniae Hrtg. (polytomus Hrtg.)]

-Neodiprion sertifer Geoffroy (Hym. Diprionidae) [48, 51]

-Gilpinia pallida Klug (Hym. Diprionidae) [2-as Lophyrus-rev. 18-as Diprion] -Neodiprion sertifer Geoffroy (Hym. Diprionidae) $[48,51,57,67]$

-Dendrolimus pini Linnaeus (Lep. Lasiocampidae) [41-rev. HE-s.a. 39]

-Lymantria monacha Linnaeus (Lep. Lymantriidae) [39-rev. HE 73-s.a. 74] -Diprion pini Linnaeus (Hym. Diprionidae) [39-rev. HE 42, 87, new rec.-CZ, Zbiroh, leg. I. Hájek, 1970, VULH-rev. HE]

-Gilpinia pallida Klug (Hym. Diprionidae) [2-as Lophyrus-rev. 18-as Diprion] -Gilpinia polytoma Hartig (Hym. Diprionidae) [15, 59-as Diprion polytomum] -Gilpinia variegata Hartig (Hym. Diprionidae) [2-as Lophyrus-rev. 18-as Diprion] -Gilpinia virens Klug (Hym. Diprionidae) [2-as Lophyrus-rev. 18-as Diprion]

-Neodiprion sertifer Geoffroy (Hym. Diprionidae) [2-as Lophyrus rufus-rev. 18, 14, 37-s.a. 38-rev. HE 48, 51, 57, 67]

-Deilephila elpenor Linnaeus (Lep. Sphingidae) [39-as Pergesa-rev. HE, 106-as Chaerocampa]

-Naenia typica Linnaeus (Lep. Noctuidae) [6]

-[Malacosoma neustria Linnaeus (Lep. Lasiocampidae)] [100-as Bombyx-s.a. 105, 108; prb. misid.]

-[Saturnia pavonia Linnaeus (Lep. Saturniidae)] [105-as carpini; host prb. wrong] -[Calliteara pudibunda Linnaeus (Lep. Lymantriidae)] [100-as Dasychira-s.a. 105, 108; prb. misid.]

-[Lymantria dispar Linnaeus (Lep. Lymantriidae)] [105; questionable host] -Spilosoma luteum Hufnagel (Lep. Arctiidae) [39-as Spilarctia lubricipeda] -Spilosoma sp. (Lep. Arctiidae) [37]

-Phragmatobia fuliginosa Linnaeus (Lep. Arctiidae) [100-as Spilosoma-s.a. 105, 108]

-Arctia caja Linnaeus (Lep. Arctiidae) [4, 39-rev. HE 63, 100-s.a. 104, 105, 108]

-Arctia villica Linnaeus (Lep. Arctiidae) [101-s.a. 105, 108]

-Arctia festiva Hufnagel (Lep. Arctiidae) [100-as hebe-s.a. 102, 104, 105, 108]

-Orgyia antiqua Linnaeus (Lep. Lymantriidae) [2, 18-rev. 19]

-[Cimbex femoratus Linnaeus (Hym. Cimbicidae)] [2, 18-both as betulae Zdd.-rev. 
Carcelia gnava (Meigen, 1824) (as Sisyropa excissa-100, S. excissa and C. gnava-104, excissa and gnava-105)

(as excissa; prb. misid.)

(as Parexorista and Sisyropa excavata100; excissa and gnava-105, 108; "excissa" is a wrong synonymization of excavata, see 103)

(as Parexorista-100; prb. misid. =prb. Carcelia lucorum)

Carcelia iliaca (Ratzeburg, 1840)

Carcelia laxifrons Villeneuve, 1912

(misid. as Parexorista cheloniae-2)

Carcelia rasa (Macquart, 1849)

Carcelia sp.

Senometopia excisa (Fallén, 1820)

(as Carcelia)

(as Sisyropa excissa -102, 104; Carcelia105 ; might be some species of

Senometopia or Carcelia)

(as Carcelia excissa; prb. misid. =prb.

Carcelia puberula)

(as Carcelia excissa; prb. misid. =prb.

Carcelia bombylans)

Senometopia pollinosa (Mesnil, 1941) (as

Carcelia obesa Zett.)

(as Carcelia rutilla Meig.)

(as Carcelia obesa Zett.-6; misid. as

"Nemorea erythrura" = prb. S. pollinosa-

17; Carcelia rutilla Meig.-37, 82; Exorista

flavicans-114)

Senometopia susurrans (Rondani, 1859)

(as Carcelia; prb. misid., identity

unknown)

Erycia fatua (Meigen, 1824)

(prb. misid. =prb. Allophorocera

ferruginea which is a parasitoid of

Tipulidae)

Erycia festinans (Meigen, 1824) (as

Hemimasicera; prb. misid. $=$ prb.

Allophorocera ferruginea which is a parasitoid of Tipulidae)

Xylotachina diluta (Meigen, 1824) (as

Lydella ambulans $\mathrm{Rd}$.)

Townsendiellomyia nidicola (Townsend, 1908)
19; host unusual for Carcelia and presumably wrong]

-Malacosoma neustria Linnaeus (Lep. Lasiocampidae) [100 -as Bombyx, 104-as Malacosoma and Bombyx, 105]

-[Proserpinus proserpina Pallas (Lep. Sphingidae)] [105-as Pterogon, s.a. 108] -[Aglais urticae Linnaeus (Lep. Nymphalidae)] [25-tachinid confirmed by TS, but host unusual for Carcelia and prb. wrong]

-Calliteara pudibunda Linnaeus (Lep. Lymantriidae) [4-as Dasychira, 87, 97, 100as Dasychira-s.a. 104, 105, 108]

-Leucoma salicis Linnaeus (Lep. Lymantriidae) [new rec.-CZ, Kaplice env., leg. Kolubajiv?, 1966, VULH-rev. HE]

-[Arctia caja Linnaeus (Lep. Arctiidae)] [100-s.a. 104, 105, 108]

-Thaumetopoea processionea Linnaeus (Lep. Thaumetopoeidae) [87]

-Leucoma salicis Linnaeus (Lep. Lymantriidae) [2-rev. 18-as Stilpnotia]

-Calliteara pudibunda Linnaeus (Lep. Lymantriidae) [63, 87]

-[Choristoneura murinana Hübner (Lep. Tortricidae)] [81-s.a. 82-as Cacoecia; prb. wrong because Carcelia does not parasitize Tortricidae]

-[Sphinx pinastri Linnaeus (Lep. Sphingidae)] [39-as Hyloicus; host prb. wrong]

-[Pheosia gnoma Fabricius (Lep. Notodontidae)] [102-as Notodonta dictoides Huf.s.a. 104, 105]

-[Lymantria monacha Linnaeus (Lep. Lymantriidae)] [108-as Limantria]

-[Phragmatobia fuliginosa Linnaeus (Lep. Arctiidae)] [108]

-[Zygaena filipendulae Linnaeus (Lep. Zygaenidae)] [6; host prb. wrong]

-[Dendrolimus pini Linnaeus (Lep. Lasiocampidae)] [105; host prb. wrong]

-Bupalus piniarius Linnaeus (Lep. Geometridae) [6, 17-as Fidonia piniaria 37, 82, 87, 114-rev.]

-[Dioryctria abietella Denis \& Schiff. (Lep. Pyralidae)] [111]

-Melitaea athalia Rottemburg (Lep. Nymphalidae) [105]

-[Lymantria monacha Linnaeus (Lep. Lymantriidae)] [74]

-[Lymantria monacha Linnaeus (Lep. Lymantriidae)] [74]

-Cossus cossus Linnaeus (Lep. Cossidae) [105]

-Euproctis chrysorrhoea Linnaeus (Lep. Lymantriidae) [new rec.-CZ, Dambořice, leg. Kolubajiv?, 1955, VULH-rev. HE] 


\section{Goniini}

Eumea linearicornis (Zetterstedt, 1844)

(as Exorista westermanni and misid. in part as Exorista mitis and Blondelia nigripes)

(misid. as Phryxe vulgaris)

(as Exorista westermanni)

(as spernanda Zett)

(as Exorista westermanni and misid. as $E$. mitis in part)

Eumea mitis (Meigen, 1824)

(as Exorista)

(as Exorista)

(as Exorista)

(as Platymyia)

(misid. as Parexorista temera Mg.-2)

Myxexoristops abietis Herting, 1964 (as M. abietis and prb. misid. $M$. blondeli-49)

Myxexoristops bicolor (Villeneuve, 1908) (as $M$. bicolor, and prb. misid. $M$. bonsdorffi and $M$. blondeli)

Myxexoristops bonsdorffi (Zetterstedt, 1859) (misid. as Parexorista cheloniae Rd.-2)

Zenillia dolosa (Meigen, 1824) (as libatrix f. grisella; ident. not confirmed) (as libatrix f. grisella; ident. not confirmed)

Zenillia libatrix (Panzer, 1798)

(as Exorista; $=$ Z. libatrix or misid.) (prb. misid. $=$ Senometopia pollinosa)

Clemelis pullata (Meigen, 1824) Pales pavida (Meigen, 1824) (misid. as Phryxe vulgaris)

(as Ctenophorocera-64)

(as Nilea-39)

(prb. misid. of Pales processioneae)

(as Nilea-39; Ctenophorocera-116) (as Nilea-39)

(as Phorocera cilipeda-2)
-Tortrix viridana Linnaeus (Lep. Tortricidae) [39-rev. HE]

-Archips rosana Linnaeus (Lep. Tortricidae) [39-as Cacoecia-rev. HE]

-Pandemis cerasana Hübner (Lep. Tortricidae) [39-as ribeana-rev. HE]

-Cosmia affinis Linnaeus (Lep. Noctuidae) [5]

-Cosmia trapezina Linnaeus (Lep. Noctuidae) [39-as Calymnia-rev. HE]

-Megalophanes viciella Denis \& Schiff. (Lep. Psychidae) [6-as Psyche]

-Archips crataegana Hübner (Lep. Tortricidae) [20-as Cacoecia-s.a. 21]

-[Dendrolimus pini Linnaeus (Lep. Lasiocampidae)] [41-s.a. 39; questionable host]

-Cosmia trapezina Linnaeus (Lep. Noctuidae) [39-rev. HE]

-Cerapteryx graminis Linnaeus (Lep. Noctuidae) [84-as Charaeas]

-Nematus oligospilus Förster (Hym. Tenthredinidae) [2-rev. 18-as Pteronidea oligospila]

-Cephalcia abietis Linnaeus (Hym. Pamphiliidae) [49-material reared from

Cephalcia sp., misid. by Čepelák as $M$. blondeli, was found by HE in coll. VULH, 52]

-Cephalcia sp. (Hym. Pamphiliidae) $[52,53]$

-Cephalcia abietis Linnaeus (Hym. Pamphiliidae) [49; material reared from Cephalcia sp., misid. by Čepelák as $M$. bonsdorffi and $M$. blondeli, was found by HE in coll. VULH]

-Cephalcia alpina Klug (Hym. Pamphiliidae) [55-as falleni]

-Cephalcia arvensis Panzer (Hym. Pamphiliidae) $[54,56]$

-Acantholyda posticalis Matsumura (Hym. Pamphiliidae) [2-as Lyda stellata-rev. 18-as A. stellata]

-Tortrix viridana Linnaeus (Lep. Tortricidae) [39]

-Euproctis chrysorrhoea Linnaeus (Lep. Lymantriidae) [39]

-Pandemis cerasana Hübner (Lep. Tortricidae) [39-as ribeana]

-Malacosoma neustria Linnaeus (Lep. Lasiocampidae) [8, 104-s.a 105, 108]

-Erannis defoliaria Clerck (Lep. Geometridae) [37-as Hibernia]

-[Bupalus piniarius Linnaeus (Lep. Geometridae)] [104-s.a. 105]

-Thaumetopoea processionea Linnaeus (Lep. Thaumetopoeidae) $[\mathbf{5}, \mathbf{8 7}]$

-Euproctis chrysorrhoea Linnaeus (Lep. Lymantriidae) [39-rev HE, 104-s.a. 105, $108]$

-Lymantria dispar Linnaeus (Lep. Lymantriidae) [87]

-Pandemis heparana Denis \& Schiff. (Lep. Tortricidae) [6]

-Zeiraphera griseana Hübner (Lep. Tortricidae) [37-as Semasia diniana-rev. HE] -Eriogaster lanestris Linnaeus (Lep. Lasiocampidae) [37-rev. HE] -Malacosoma neustria Linnaeus (Lep. Lasiocampidae) [64, 104-s.a. 105, new rec.CZ, Praha-Hrdlořezy, leg. Kolubajiv, 1937, VULH-rev. HE] -Dendrolimus pini Linnaeus (Lep. Lasiocampidae) [41-s.a. 39, 87] -Thaumetopoea processionea Linnaeus (Lep. Thaumetopoeidae) [5] -Panolis flammea Denis \& Schiff. (Lep. Noctuidae) [37-rev. HE] -Autographa gamma Linnaeus (Lep. Noctuidae) [68-as Plusia-s.a. 69] -Euproctis chrysorrhoea Linnaeus (Lep. Lymantriidae) [4, 39, 116-as phaeorrhoea] -Leucoma salicis Linnaeus (Lep. Lymantriidae) [26-as Stilpnotia-s.a. 32, 39-rev. HE, new rec.-CZ, Kaplice, leg. Kolubajiv, 1966, VULH-rev. HE] -Lymantria monacha Linnaeus (Lep. Lymantriidae) [26-as Liparis-s.a. 27, 32] -[Allantus truncatus Klug (Hym. Tenthredinidae)] [2-as Emphytus cingillum-rev. 18; 
Pales pumicata (Meigen, 1824) (as Nilea; must be misid., identity unknown) (as Nilea; must be misid., identity unknown)

(as Nilea; must be misid., identity unknown)

Phryno vetula (Meigen, 1824)

Cyzenis albicans (Fallén, 1810)

Bothria frontosa (Meigen, 1824)

Ceromasia rubrifrons (Macquart, 1834)

(as florum Macq.)

(misid. as Masicera rutila Mg.-100;

Ceromasia (Masicera) ferruginea $\mathrm{Mg} .=$ florum Rd.-104; florum Macq. and Erycia fatua Mg.-105)

(as florum Macq.; prb. misid. =prb. Allophorocera ferruginea)

Allophorocera ferruginea (Meigen, 1824) (misid. as Ceromasia rutilla Mg.; $=$ prb. A. ferruginea which is a parasitoid of Tipulidae)

(as Ceromasia ferruginea Fall.) Allophorocera rufipes (Brauer \& Bergenstamm, 1891) (as Tachina vicinalis Pand.; possibly misid.)

Rhacodinella apicata (Pandellé, 1896) Eurysthaea scutellaris (RobineauDesvoidy, 1848)

Elodia morio (Fallén, 1820)(as Elodia tragica and misid. Degeeria luctuosa) Sturmia bella (Meigen, 1824)

Blepharipa pratensis (Meigen, 1824) (as Blepharipoda scutellata-39; Sturmia scutellata-41)

(as Sturmia scutellata-104; S. scutellata and Blepharipoda major-105; $S$.

scutellata-111; possibly misid. Drino galii)

(as Sturmia scutellata; prb. misid. =prb. Sturmia bella)

(as Sturmia scutellata)

Masicera pavoniae (Robineau-Desvoidy, 1830) (misid. as sylvatica-13; pratensis Meig.-18, 104, 105; Sturmia scutellata$107=$ prb. misid.) tachinid confirmed by TS, but prb. wrong]

-[Paranthrene tabaniformis Rottemburg (Lep. Sesiidae)] [39]

-[Saperda carcharias Linnaeus (Col., Cerambycidae)] [39]

-[Diprion pini Linnaeus (Hym. Diprionidae)] [39]

-[Lymantria monacha Linnaeus (Lep. Lymantriidae)] [74-s.a. 108; questionable host]

-Operophtera brumata Linnaeus (Lep. Geometridae) [4, 61]

-[Aglais urticae Linnaeus (Lep. Nymphalidae)] [105-as Vanessa; questionable because hosts of Bothria are Noctuidae]

-Zygaena carniolica Scopoli (Lep. Zygaenidae) [109]

-Zygaena sp. (Lep. Zygaenidae) [100-as fausta-s.a. 104, 105; Z. fausta is unknown from $\mathrm{CZ}$, so it was prb. a misid. of some other Zygaena]

-[Lymantria monacha Linnaeus (Lep. Lymantriidae)] [37, 39, 105; hosts prb. larvae of Tipulidae in soil samples]

-[Dendrolimus pini Linnaeus (Lep. Lasiocampidae)] [41-s.a. 39]

-[Lymantria monacha Linnaeus (Lep. Lymantriidae)] [86; host prb. wrong]

-[Lymantria monacha Linnaeus (Lep. Lymantriidae)] [105; host prb. wrong]

-Phyllodesma ilicifolia Linnaeus (Lep. Lasiocampidae) [63]

-Tortrix viridana Linnaeus (Lep. Tortricidae) [new rec.-CZ, Śárka, leg. Kolubajiv?, 1946, VULH-rev. HE]

-Tortrix viridana Linnaeus (Lep. Tortricidae) [39-rev. HE]

-Nymphalis polychloros Linnaeus (Lep. Nymphalidae) [37-as Vanessa]

-Inachis io Linnaeus (Lep. Nymphalidae) [4-as Vanessa jo 6-as Nymphalis-s.a. 116,

25]

-Aglais urticae Linnaeus (Lep. Nymphalidae) [4-as Vanessa, new rec.-CZ, Moravia, Herálec, 2003, leg., det. and coll. P. Mückstein]

-Vanessa atalanta Linnaeus (Lep. Nymphalidae) [6, new rec.-CZ, Bohemia or.,

Rovný env., 2003, leg., det. and coll. P. Mückstein]

-[Phalera bucephala Linnaeus (Lep. Notodontidae)] [4; doubtful host]

-[Leucoma salicis Linnaeus (Lep. Lymantriidae)] [26-as Stilpnotia-s.a. 32; doubtful host]

-Dendrolimus pini Linnaeus (Lep. Lasiocampidae) [41-rev. HE-s.a. 39]

-Hyles euphorbiae Linnaeus (Lep. Sphingidae) [104-as Deilephila-s.a. 105, 111]

-[Aglais urticae Linnaeus (Lep. Nymphalidae)] [105-as Vanessa]

-[Lymantria monacha Linnaeus (Lep. Lymantriidae)] [74, 105; questionable host]

-Saturnia pyri Denis \& Schiff. (Lep. Saturniidae) [2-rev. 18; "R. v. Stein” on label 
(as pratensis)

(as pratensis $\mathrm{Mg}$.)

Masicera silvatica (Fallén, 1810)

(=silvatica or misid.)

Masicera sphingivora (RobineauDesvoidy, 1830) (as pratensis-100, 104, 105; silvatica -103 ; prb. misid. $=$ prb. sphingivora)

(as pratensis; prb. misid. $=$ prb. sphingivora)

(as silvatia -105 ; prb. misid. $=$ prb. sphingivora)

Prosopea nigricans (Egger, 1861) (prb. misid., identity unknown)

Frontina laeta (Meigen, 1824)

Thelymorpha marmorata (Fabricius, 1805 ) (as vertiginosa Fll.-2, as Histochaeta-6, 18)

Baumhaueria goniaeformis (Meigen, 1824) (possibly misid.)

Gonia capitata (De Geer, 1776) (as capitata and divisa-105; divisa Mg.68,$70 ;$ prb. misid. =prb. capitata) Gonia picea (Robineau-Desvoidy, 1830) (as Salmacia sicula R.-D.)

Spallanzania hebes (Fallén, 1820)

(as Pseudogonia-70; Cnephalia-105)

\section{Subfamily Tachininae}

\section{Tachinini}

Tachina grossa (Linnaeus, 1758)

(as Echinomyia)

Tachina fera (Linnaeus, 1761)(as

Echinomyia)

(as Echinomyia-18)

(as Echinomyia; $=T$. fera or misid. $T$. magnicornis)

Peleteria ferina (Zetterstedt, 1844) (as Tachina-100; Echinomya-105)

Peleteria rubescens (Robineau-Desvoidy, 1830) (as Pallatiera prompta Mg.-74 = misid. P. rubescens; nigricornis-105)

\section{Nemoraeini}

Nemoraea pellucida (Meigen, 1824)

(as rubrica Mg.-106; rubica-107)

\section{Linnaemyiini}

Linnaemya comta (Fallén, 1810) (as

Micropalpus comptus-101, 102; compta-104, 105)

Linnaemya impudica (Rondani, 1859)

(as Micropalpus impudicus) according to HE, 13, 104-s.a. 105, 107]

-Saturnia spini Denis \& Schiff. (Lep. Saturniidae) [104-s.a. 105]

-Saturnia pavonia Linnaeus (Lep. Saturniidae) [99-as carpini 100-s.a. 104]

-Lasiocampa trifolii Denis \& Schiff. (Lep. Lasiocampidae) [105]

-Macrothylacia rubi Linnaeus (Lep. Lasiocampidae) [4]

-Smerinthus ocellatus Linnaeus (Lep. Sphingidae) [100-s.a. 103, 104, 105]

-Laothoe populi Linnaeus (Lep. Sphingidae) [104-as Smerinthus-s.a. 105]

-Hyles euphorbiae Linnaeus (Lep. Sphingidae) [98, 105-as Deilephila]

-[Ceramica pisi Linnaeus (Lep. Noctuidae)] [105-as Mamestra]

-Sphinx ligustri Linnaeus (Lep. Sphingidae) [105]

-Smerinthus ocellatus Linnaeus (Lep. Sphingidae) [105-as ocellata]

-Arctia caja Linnaeus (Lep. Arctiidae) [2-rev. 18, 6]

-Shargacucullia scrophulariae Denis \& Schiff. (Lep. Noctuidae) [105-as Cucullia] -Euproctis chrysorrhoea Linnaeus (Lep. Lymantriidae) [10-as phaeorrhoea]

-Agrotis segetum Denis \& Schiff. (Lep. Noctuidae) [68-s.a. 70, 105]

-Cerapteryx graminis Linnaeus (Lep. Noctuidae) [84, 85-s.a. 116-as Chareas]

-Agrotis segetum Denis \& Schiff. (Lep. Noctuidae) [70-s.a. 105]

-Lasiocampa trifolii Denis \& Schiff. (Lep. Lasiocampidae) [105]

-Lithomoia solidaginis Hübner (Lep. Noctuidae) [107-as Catocala]

-Ceramica pisi Linnaeus (Lep. Noctuidae) [2-as Mamestra-rev. 18]

-Lymantria monacha Linnaeus (Lep. Lymantriidae) [73-s.a. 74]

-Parasemia plantaginis Linnaeus (Lep. Arctiidae) [100-as Nemeophila-s.a. 105]

-[Lymantria monacha Linnaeus (Lep. Lymantriidae)] [74, 105; host prb. wrong]

-Colocasia coryli Linnaeus (Lep. Noctuidae) [106-as Demas-s.a. 107]

-[Saturnia pavonia Linnaeus (Lep. Saturniidae)] [101-as carpini-s.a. 102, 104, 105; doubtful because Linnaemya is not known as a parasitoid of the very commonly reared genus Saturnia]

-Agrotis sp. (Lep. Noctuidae) [107] 
(as Micropalpus impudicus)

Linnaemya haemorrhoidalis (Fallén, 1810) (as Micropalpus-102)

Linnaemya picta (Meigen, 1824)

(as Micropalpus pictus Mg.; = picta or Linnaemya sp.)

Lypha dubia (Fallén, 1810)

\section{Ernestiini}

Ernestia rudis (Fallén, 1810) (as Panzeria strenua $M g . ;=E$. rudis or misid.)

(as Panzeria strenua $M g . ;=E$. rudis or misid.)

Eurithia anthophila (RobineauDesvoidy, 1830) (as Erigone radicum Fll.-2; Ernestia radicum F.-18) Eurithia connivens (Zetterstedt, 1844) (as Ernestia)

Eurithia consobrina (Meigen, 1824) (as Ernestia; prb. misid., identity unknown) (as Ernestia)

(as Ernestina)

Hyalurgus tomostethi Čepelák, 1963

Cleonice callida (Meigen, 1824)

Cleonice keteli Ziegler, 2000 (misid. as callida)

\section{Brachymerini}

Pseudopachystylum goniaeoides

(Zetterstedt, 1838)

\section{Pelatachinini}

Pelatachina tibialis (Fallén, 1810)

(as Hyria)

\section{Macquartiini}

Anthomyiopsis plagioderae Mesnil, 1972

(misid. as A. nigrisquamata)

Triarthriini

Triarthria setipennis (Fallén, 1810)

(as Bigonichaeta)
-[Euproctis chrysorrhoea Linnaeus (Lep. Lymantriidae)] [39; host prb. wrong]

-[Saturnia pavonia Linnaeus (Lep. Saturniidae)] [102-s.a. 104-as pavoniae and carpini, 105-as carpini; doubtful, see note under Linnaemya comta]

-[Hyles euphorbiae Linnaeus (Lep. Sphingidae)] [105-as

Deilephila; questionable because hosts of $L$. picta are Noctuidae]

-Retinia resinella Linnaeus (Lep. Tortricidae) [36]

-Rhyacionia buoliana Denis \& Schiff. (Lep. Tortricidae) [50]

-Operophtera brumata Linnaeus (Lep. Geometridae) [61]

-[Saturnia pyri Denis \& Schiff. (Lep. Saturniidae)] [105; host prb. wrong]

-[Saturnia pavonia Linnaeus (Lep. Saturniidae)] [105-as carpini; host prb. wrong] -Panolis flammea Denis \& Schiff. (Lep. Noctuidae) [37]

-[Lymantria monacha Linnaeus (Lep. Lymantriidae)] [37, 74, 105; ident. in part confirmed by $\mathrm{HE}$, but host must be wrong because $E$. rudis failed to parasitize $L$. monacha in rearing experiments]

-Spilosoma lubricipeda Linnaeus (Lep. Arctiidae) [2-as menthastri-rev. 18]

-[Choristoneura murinana Hübner (Lep. Tortricidae)] [81-s.a. 82-as Cacoecia; tachinid ident. correct (det. Mesnil), but host too small for Eurithia]

-[Eriogaster lanestris Linnaeus (Lep. Lasiocampidae)] [37]

-Cucullia artemisiae Hufnagel (Lep. Noctuidae) [105]

-[Lymantria dispar Linnaeus (Lep. Lymantriidae)] [105; host prb. wrong]

-Tomostethus nigritus Fabricius (Hym. Tenthredinidae) [9-s.a. 60]

-Chrysomela populi Linnaeus (Col. Chrysomelidae) [95]

-Chrysomela vigintipunctata Scopoli (Col. Chrysomelidae) [91-rev. TS]

-Cephalcia arvensis Panzer (Hym. Pamphiliidae) [54]

-Cephalcia sp. (Hym. Pamphiliidae) [49]

-Nymphalis antiopa Linnaeus (Lep. Nymphalidae) [26-as Vanessa-s.a. 30, 32] -Inachis io Linnaeus (Lep. Nymphalidae) [26-as Vanessa-s.a. 30, 32]

-Lacanobia oleracea Linnaeus (Lep. Noctuidae) [2-as Mamestra; untypical host]

-[Altica quercetorum Foudras (Col. Chrysomelidae)] [89-rev. VA, TS; the host is unlikely because $A$. plagioderae is known from Chrysomelidae on Salix, primarily Plagiodera versicolora; first record of $A$. plagioderae for $\mathbf{C Z}]$

-Forficula auricularia Linnaeus (Derm. Forficulidae) [new rec.-CZ, Žatec (=Saaz), NHMW-rev. TS]

-[Gilpinia polytoma Hartig (Hym. Diprionidae)] [39; host must be wrong]

\section{Neaerini}

Phytomyptera cingulata (Robineau- 
Desvoidy, 1830)

Phytomyptera nigrina (Meigen, 1824)

Graphogaster brunnescens Villeneuve, 1907

\section{Siphonini}

Ceromya bicolor (Meigen, 1824)

(as Actia)

Ceromya flaviceps (Ratzeburg, 1844) (as Actia-6, 39)

Actia crassicornis (Meigen, 1824)

Actia resinellae (Schrank, 1781) (as nudibasis Stein-43)

(as nudibasis Stein-12,36; misid. as pilipennis-39)

(as nudibasis St.-29, 30, 32, 36, 50; misid. as pilipennis-39; misid. as Tryptocera crassicornis-113)

Actia pilipennis (Fallén, 1810)

Siphona sp. (as Bucentes cristata; cristata is prb. correct but not yet confirmed) (as cristata incl, flavifrons $-18=\mathrm{prb}$. cristata)

(as Bucentes geniculata; prb. misid. = prb. S. cristata)

\section{Leskiini}

Demoticus plebejus (Fallén, 1810) (prb. misid., identity unknown)

Leskia aurea (Fallén, 1820)
-Tineidae sp. (ex Carpinus betulus) (Lep. Tineidae) [87]

-[Anthonomus piri Kollar (Col. Curculionidae)] [39-as pyri; tachinid rev. by HE, but host must be wrong]

-Acleris ferrugana Denis \& Schiff. (Lep. Tortricidae) [18]

-Lasiocampa quercus Linnaeus (Lep. Lasiocampidae) [71, 72]

-Dendrolimus pini Linnaeus (Lep. Lasiocampidae) [6-s.a. 39, 87]

-Cosmotriche lobulina Denis \& Schiff. (Lep. Lasiocampidae) [87]

-Depressaria daucella Denis \& Schiff. (Lep. Oecophoridae) [78-as nervosa]

-Exoteleia dodecella Linnaeus (Lep. Gelechiidae) [43]

-Retinia resinella Linnaeus (Lep. Tortricidae) [36-s.a. 12, 39-as Evetria-rev. HE, new rec.-CZ, no locality, 1936, VULH-rev. HE]

-Rhyacionia buoliana Denis \& Schiff. (Lep. Tortricidae) [29-as Evetria-s.a. 30-as Grapholitha and 32-as Evetria, 36-s.a. 12, 39-rev. HE, 50, 113-as Retinia-rev. 87] -Tortrix viridana Linnaeus (Lep. Tortricidae) [39-rev. HE]

-Archips xylosteana Linnaeus (Lep. Tortricidae) [39-as Cacoecia]

-Autographa gamma Linnaeus (Lep. Noctuidae) [69-as Plusia]

-Ceramica pisi Linnaeus (Lep. Noctuidae) [2-as Mamestra-rev. 18]

-Mamestra brassicae Linnaeus (Lep. Noctuidae) [105]

-[Arctia festiva Hufnagel (Lep. Arctiidae)] [105-as hebe]

-Synanthedon vespiformis Linnaeus (Lep. Sesiidae) [100-as Sesia asiliformis-s.a. 103-as Sesia asiliformis and cynipiformis, 105-as Sesia new rec.-CZ, Závist near Černá Hora, 1977 and Maršovice near Moravský Krumlov, 1993, leg. and det. Laštůvka, pers. comm.]

-Synanthedon myopaeformis Borkhausen (Lep. Sesiidae) [new rec.-CZ, Brno-Nový Lískovec, 2006, leg. Šefrová, det. Laštůvka, pers. comm.]

\section{Microphthalmini}

Dexiosoma caninum (Fabricius, 1781)

(possibly misid. Dexia rustica)

-Melolontha melolontha Linnaeus (Col. Scarabaeidae) [39]

\section{Subfamily Dexiinae}

\section{Dexiini}

Billaea adelpha (Loew, 1873) (ident. not confirmed)

Billaea irrorata (Meigen, 1826)

-Isarthron fuscum Fabricius (Col. Cerambycidae) [82-as Tetropium]

(as Phorostoma parvula Portsch.-114)

-Oberea pupillata Gyllenhal (Col. Cerambycidae) [4, 33-s.a.]

Billaea triangulifera (Zetterstedt, 1844)

(as Gymnodexia)

-Saperda populnea Linnaeus (Col. Cerambycidae) [4, 87, 114-rev.]

-Leiopus nebulosus Linnaeus (Col. Cerambycidae) [4-as Liopus]

- Oplosia fennica Paykull (Col. Cerambycidae) [24]

-Saperda octopunctata Scopoli (Col. Cerambycidae) [7]

(as Gymnodexia)

-[Saperda populnea Linnaeus (Col. Cerambycidae)] [7, 103; doubtful because the well-known parasitoid of this host is Billaea irrorata]

Dexia rustica (Fabricius, 1775) (as Dixia) -Melolontha melolontha Linnaeus (Col. Scarabaeidae) [115] 


\section{Voriini}

Peteina erinaceus (Fabricius, 1794) Athrycia impressa (Wulp, 1869) (misid. as Plagia ruralis-2)

Voria ruralis (Fallén, 1810)

(as Plagia-2)

Hyleorus elatus (Meigen, 1838) (as

Athrycia elata)

Phenicellia haematodes (Meigen, 1824)

(as Phoenicella)

Thelaira nigripes (Fabricius, 1794)

Thelaira sp. (as leucozona Panz.; older species identifications of Thelaira are unreliable)

-Autographa gamma Linnaeus (Lep. Noctuidae) [2-as Plusia-rev. 18]

-Anarta myrtilli Linnaeus (Lep. Noctuidae) [2-rev. 18]

-Autographa gamma Linnaeus (Lep. Noctuidae) [4-as Plusia, 105-as Plusia]

-Arctia festiva Hufnagel (Lep. Arctiidae) [2-as hebe-rev. 18]

-Euproctis similis Fuessly (Lep. Lymantriidae) [6-as Porthesia]

-Arctia festiva Hufnagel (Lep. Arctiidae) [6-as hebe]

-Abrostola asclepiadis Denis \& Schiff. (Lep. Noctuidae) [62]

-Laothoe populi Linnaeus (Lep. Sphingidae) [101-as Smerinthus-s.a. 105]

(as leucozona Panz.-101; nigripes F.-105) -Deilephila elpenor Linnaeus (Lep. Sphingidae) [101, s.a. 105]

(as leucozona Panz.)

-[Lymantria monacha Linnaeus (Lep. Lymantriidae)] [74, 105; questionable host]

(as leucozona Panz.; possibly correct in this case because the true leucozona is a parasitoid of Arctia caja)

Rondania dimidiata (Meigen, 1824)

-Arctia caja Linnaeus (Lep. Arctiidae) [105]

-[Zeiraphera rufimitrana Herrich-Schäffer (Lep. Tortricidae)] [87-tachinid confirmed, but host must be wrong]

(misid. as Erynnia nitida-rev. HE)

-[Lymantria monacha Linnaeus (Lep. Lymantriidae)] [37-host must be wrong] -Liparus sp. (Col. Curculionidae) [39-as Molytes]

\section{Dufouriini}

Microsoma exiguum (Meigen, 1824) (as Syntomogaster exigua)

-[Tortrix viridana Linnaeus (Lep. Tortricidae)] [39-tachinid confirmed by HE, but host must be wrong]

\section{Host-parasitoid list}

$\begin{array}{ll}\text { Lepidoptera } & \\ \text { Tineidae } & \text {-Tineidae g. sp. } \\ \text { Psychidae } & \text {-Megalophanes viciella } \\ \text { Yponomeutidae } & \text {-Yponomeuta evonymella } \\ & \text {-Yponomeuta padella } \\ \text { Gelechiidae } & \text {-Exoteleia dodecella } \\ \text { Oecophoridae } & \text {-Depressaria daucella } \\ \text { Zygaenidae } & \text {-Zygaena carniolica } \\ & \text {-Zygaena ephialtes } \\ & \text {-Zygaena angelicae } \\ & \text {-Zygaena filipendulae } \\ & \text {-Zygaena laeta } \\ & \text {-Zygaena punctum } \\ & \text {-Zygaena sp. } \\ \text { Sesiidae } & \text {-Paranthrene tabaniformis } \\ & \text {-Synanthedon vespiformis } \\ & \text {-Synanthedon myopaeformis } \\ \text { Cossidae } & \text {-Cossus cossus } \\ \text { Tortricidae } & \text {-Cochylimorpha hilarana } \\ & \text {-Tortrix viridana }\end{array}$

- Acleris ferrugana

- Archips crataegana

- Archips xylosteana

- Archips rosana
-Phytomyptera cingulata

-Eumea mitis

-Bessa parallela, Blondelia nigripes

-Bessa parallela, Blondelia nigripes, ${ }^{*}$ Phryxe vulgaris

- Actia resinellae

-Actia crassicornis

-Phryxe prima, Ceromasia rubrifrons

-Exorista larvarum

-Phryxe setifacies

-[Senometopia pollinosa $]$

-Phryxe magnicornis

-Phryxe prima, Exorista larvarum

-Ceromasia rubrifrons, Exorista larvarum

-[Blondelia nigripes], [Pales pumicata]

-Leskia aurea

-Leskia aurea

-Xylotachina diluta

-Blondelia nigripes

- Actia pilipennis, Elodia morio, Bessa parallela, Nemorilla floralis, Blondelia nigripes, Eumea linearicornis, Pseudoperichaeta nigrolineata, Eurysthaea scutellaris, Zenillia dolosa, Phryxe magnicornis, [Acemya acuticornis], [Microsoma exiguum]

-Bessa parallela, Graphogaster brunnescens

- Pseudoperichaeta nigrolineata, Eumea mitis

- Actia pilipennis

-Eumea linearicornis 


$\begin{array}{cl} & \text {-Choristoneura murinana } \\ & \\ & \text {-Pandemis cerasana } \\ & \text {-Pandemis heparana } \\ & \text {-Epinotia tedella } \\ & \text {-Zeiraphera rufimitrana } \\ & \text {-Zeiraphera griseana } \\ & \text {-Retinia resinella } \\ & \text {-Rhyacionia buoliana } \\ \text { Pyralidae } & \text {-Dioryctria abietella } \\ & \text {-Trachycera suavella } \\ & \text {-Ostrinia nubilalis } \\ \text { Lasiocampidae } & \text {-Eriogaster lanestris } \\ & \text { - Malacosoma neustria }\end{array}$

Bombycidae Saturniidae

Sphingidae

Papilionidae

Pieridae

Lycaenidae

Nymphalidae
-Lasiocampa trifolii - Lasiocampa quercus -Macrothylacia rubi -Dendrolimus pini

\section{-Euthrix potatoria} -Cosmotriche lobulina -Phyllodesma ilicifolia -Bombyx mori -Saturnia pyri -Saturnia spini -Saturnia pavonia

-Sphinx ligustri -Sphinx pinastri -Mimas tiliae -Smerinthus ocellatus -Laothoe populi -Macroglossum stellatarum -Proserpinus proserpina -Hyles euphorbiae

-Deilephila elpenor

-Deilephila porcellus -Zerynthia polyxena -Iphiclides podalirius - Papilio machaon - Aporia crataegi -Pieris brassicae

-Pieris rapae

-Pieris napi - Anthocharis cardamines -Neozephyrus quercus -Satyrium pruni -Callophrys rubi -Nymphalis polychloros - Nymphalis xanthomelas -Nymphalis antiopa

-Inachis io
-Nemorilla maculosa, Blondelia nigripes, Phryxe nemea, [Phebellia triseta], [Carcelia sp.], [Eurithia connivens]

-Eumea linearicornis, Zenillia libatrix, Blondelia nigripes

-Clemelis pullata

-[Admontia grandicornis]

-[Rondania dimidiata]

-Nemorilla maculosa, Blondelia nigripes, Pales pavida

- Actia resinellae, Lypha dubia

-Actia resinellae, Lypha dubia, [Acemya acuticornis]

-[Winthemia rufiventris], [Senometopia susurrans]

-Nemorilla maculosa

-Lydella thompsoni

-Pales pavida, [Eurithia consobrina]

- Carcelia gnava, Pales pavida, Compsilura concinnata, Exorista larvarum, Blondelia nigripes, Zenillia libatrix, Phryxe vulgaris, Phorocera assimilis, [Phorocera obscura], [Pseudoperichaeta nigrolineata], [Hubneria affinis]

- Tachina grossa, Masicera silvatica, Phryxe vulgaris

-Ceromya bicolor

- Masicera silvatica

-Drino inconspicua, Blepharipa pratensis, Pales pavida, Blondelia nigripes, Ceromya flaviceps, [Senometopia pollinosa], [Eumea mitis], [Allophorocera ferruginea]

-Exorista larvarum

-Exorista larvarum, Ceromya flaviceps

- Rhacodinella apicata

- Compsilura concinnata

-Masicera pavoniae, [Ernestia rudis]

-Masicera pavoniae

-Exorista grandis, Masicera pavoniae, [Exorista deligata], [Hubneria affinis], [Linnaemya comta], [Linnaemya haemorrhoidalis],

[Ernestia rudis]

-Winthemia cruentata, Compsilura concinnata, Frontina laeta

-Phryxe erythrostoma, [Senometopia excisa]

- Winthemia cruentata

-Frontina laeta, Masicera sphingivora

-Masicera sphingivora, Thelaira sp.

-Exorista larvarum

-[Carcelia gnava]

-Masicera sphingivora, Exorista grandis, *Blepharipa pratensis,

[Linnaemya picta]

-Drino lota, Winthemia quadripustulata, Exorista grandis,

Thelaira sp.

-Phryxe vulgaris

- Winthemia quadripustulata

- Compsilura concinnata

-Buquetia musca

-Phryxe vulgaris, *Aplomya confinis

-Compsilura concinnata, Phryxe vulgaris, Phryxe nemea

-Compsilura concinnata

- Compsilura concinnata, Phryxe vulgaris

-Phryxe vulgaris

-Aplomya confinis, Phryxe nemea

-Phryxe vulgaris

-Cadurciella tritaeniata

-Sturmia bella, Phryxe vulgaris, [Exorista rustica]

- Phryxe vulgaris

-Pelatachina tibialis, Compsilura concinnata, Phryxe vulgaris, Exorista larvarum

-Sturmia bella, Pelatachina tibialis, Compsilura concinnata, Phryxe 


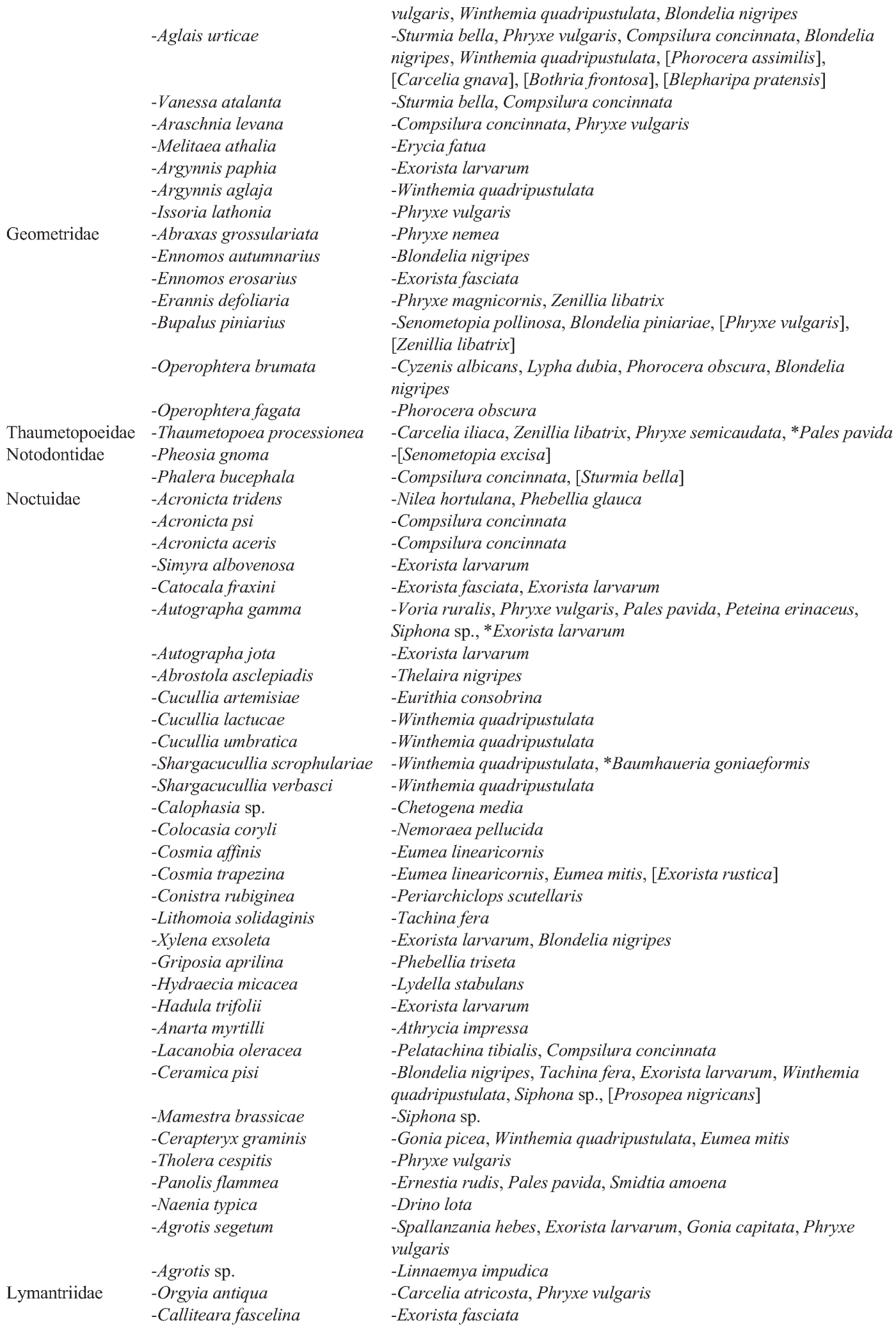

-Aglais urticae

- Vanessa atalanta

- Araschnia levana

- Melitaea athalia

-Argynnis paphia

- Argynnis aglaja

-Erannis defoliaria

Operophtera fagata

- Acronicta tridens

-Autographa gamma

-Autographa jota

- Abrostola asclepiadis

-Shargacucullia scrophulariae

-Shargacucullia verbasci

Calophasia sp.

Colocasia coryli

Cosmia affinis

Cosmia trapezina

Conistra rubiginea

-Xylena exsoleta

Griposia aprilina

raecia micacea

- Lacanobia oleracea

- Mamestra brassicae

Cerapteryx gramini.

ia typica

- Orgyia antiqua

- Calliteara fascelina vulgaris, Winthemia quadripustulata, Blondelia nigripes

-Sturmia bella, Compsilura concinnata

-Exorista larvarum

-Winthemia quadripustulata

Phryxe vulgaris

-Phryxe magnicornis, Zenillia libatrix

-Senometopia pollinosa, Blondelia piniariae, [Phryxe vulgaris]

-Cyzenis albicans, Lypha dubia, Phorocera obscura, Blondelia

nigripes

-Carcelia iliaca, Zenillia libatrix, Phryxe semicaudata, ${ }^{*}$ Pales pavida

- Senometopia excisa]

- Nilea hortulana, Phebellia glauca

- Compsilura concinnata

concinnate

ista larvarum

Siphona sp., *Exorista larvarum

- Winthemia quadripustulata

- Winthemia quadripustulato

- Chetogena media

-Eumea linearicornis, Eumea mitis, [Exorista rustica]

-Periarchiclops scutellaris

- Tachina fera

-Phebellia triseta

-Lydella stabulans

-Exorista larvarum

-Blondelia nigripes, Tachina fera, Exorista larvarum, Winthemia quadripustulata, Siphona sp., [Prosopea nigricans]

-Siphona sp.

-Gonia picea, Winthemia quadripustulata, Eumea mitis

-Drino lota

-Spallanzania hebes, Exorista larvarum, Gonia capitata, Phryxe

-Linnaemya impudica

-Exorista fasciata 
-Calliteara pudibunda

-Euproctis chrysorrhoea

-Euproctis similis

-Leucoma salicis

-Lymantria monacha

Arctiidae

\section{Coleoptera}

Carabidae

Scarabaeidae

Cerambycidae

Chrysomelidae

Curculionidae

\section{Hymenoptera}

Argidae

Cimbicidae

Diprionidae
-Lymantria dispar

- Parasemia plantaginis

-Spilosoma lubricipeda

-Spilosoma luteum

-Spilosoma sp.

- Phragmatobia fuliginosa

- Arctia caja

- Arctia villica

-Arctia festiva

-Zabrus tenebrioides

- Melolontha melolontha

-Isarthron fuscum

- Oplosia fennica

-Leiopus nebulosus

-Saperda octopunctata

-Saperda populnea

-Saperda carcharias

- Oberea pupillata

- Gastrophysa viridula

- Plagiodera versicolora

-Chrysomela populi

-Chrysomela vigintipunctata

-Linaeidea aenea

- Gonioctena quinquepunctata

- Galerucella lineola

-Lochmaea capreae

- Agelastica alni

- Altica quercetorum

-Anthonomus piri

-Liparus sp.

-Arge sp.

- Abia sericea

- Cimbex femoratus

-Cimbex sp.

- Pseudoclavellaria amerinae

-Diprion pini
- Carcelia gnava, Carcelia rasa, Compsilura concinnata,

[Hubneria affinis]

-Townsendiellomyia nidicola, Compsilura concinnata, Pales pavida, Exorista larvarum, Zenillia libatrix, Zenillia dolosa, Baumhaueria goniaeformis, [Pseudoperichaeta nigrolineata], [Linnaemya impudica]

-Hyleorus elatus

-Carcelia gnava, Exorista larvarum, Pales pavida, Carcelia laxifrons, [Exorista mimula], [Sturmia bella]

-Parasetigena silvestris, Pales pavida, Exorista larvarum, Drino inconspicua, Compsilura concinnata, Tachina fera, Exorista grandis, [Exorista rustica], [Phorocera assimilis], [Phorocera obscura], [Senometopia excisa], [Erycia fatua], [Erycia festinans], [Phryno vetula], [Ceromasia rubrifrons], [Allophorocera

ferruginea], [Allophorocera rufipes], [Blepharipa pratensis], [Peleteria rubescens], [Ernestia rudis], [Thelaira sp.], [Rondania dimidiata]

-Zenillia libatrix, [Hubneria affinis], [Eurithia consobrina]

-Peleteria ferina, [Phryxe vulgaris]

-Eurithia anthophila

-Hubneria affinis

-Hubneria affinis

-Hubneria affinis, Exorista larvarum, [Senometopia excisa]

-Hubneria affinis, Thelymorpha marmorata, Thelaira sp., Blondelia nigripes, Exorista larvarum, [Phryxe vulgaris], [Carcelia gnava]

-Hubneria affinis

-Phenicellia haematodes, Hubneria affinis, Exorista larvarum, Voria ruralis, [Phorocera assimilis], [Phorocera obscura], [Demoticus plebejus]

-Zaira cinerea

-Dexia rustica, *Dexiosoma caninum

- Billaea adelpha

-Billaea triangulifera

- Billaea triangulifera

- Billaea triangulifera

-Billaea irrorata, [Billaea triangulifera]

-[Pales pumicata]

- Billaea irrorata

-Meigenia mutabilis

-[Medina luctuosa], [Medina melania]

-Cleonice callida

-Cleonice keteli

-[Medina luctuosa]

-[Medina luctuosa]

- Medina collaris

-Medina collaris

-*Meigenia mutabilis, [Meigenia incana], [Leiophora innoxia]

-[Anthomyiopsis plagioderae]

-[Phytomyptera nigrina $]$

-Rondania dimidiata

\author{
-Belida angelicae \\ -Phebellia glirina \\ -[Carcelia atricosta] \\ - Compsilura concinnata \\ -Phebellia clavellariae \\ -Drino inconspicua, Diplostichus janitrix, Blondelia inclusa,
}




\author{
-Diprion similis \\ -Gilpinia frutetorum \\ - Gilpinia hercyniae \\ - Gilpinia pallida \\ -Gilpinia polytoma \\ -Gilpinia variegata \\ -Gilpinia virens \\ -Monoctenus juniperi \\ -Neodiprion sertifer \\ Pamphiliidae -Acantholyda posticalis \\ - Cephalcia abietis \\ - Cephalcia alpina \\ -Cephalcia arvensis \\ -Cephalcia sp. \\ Tenthredinidae -Allantus truncatus \\ -Cladius pallipes \\ -Cladius pectinicornis \\ -Hemichroa crocea \\ -Nematus melanaspis \\ - Nematus oligospilus \\ - Pristiphora abietina \\ -Pristiphora erichsonii \\ - Pristiphora pallidiventris \\ -Rhogogaster viridis \\ -Tenthredo arcuata \\ - Tenthredopsis coquebertii \\ -Tenthredopsis scutellaris \\ -Tomostethus nigritus \\ -Trichiocampus grandis
}

[Exorista mimula], [Pales pumicata]

-Diplostichus janitrix

-Diplostichus janitrix

-Diplostichus janitrix, Drino bohemica

-Drino gilva, Drino inconspicua

-Drino inconspicua, Bessa selecta, Blondelia inclusa, Diplostichus

janitrix, Blondelia nigripes, [Triarthria setipennis]

-Diplostichus janitrix, Drino inconspicua

-Drino inconspicua, Diplostichus janitrix

-Staurochaeta albocingulata

-Drino inconspicua, Blondelia inclusa, Drino gilva, Drino bohemica

-Myxexoristops bonsdorffi

-Myxexoristops abietis, Myxexoristops bicolor, [Nilea hortulana $]$

-Myxexoristops bicolor

-Myxexoristops bicolor, Pseudopachystylum goniaeoides

-Myxexoristops abietis, Pseudopachystylum goniaeoides

-[Pales pavida]

- Bessa selecta

-Exorista mimula

- Bessa selecta

-Bessa selecta

-Eumea mitis

-[Bactromyia aurulenta]

- Bessa selecta

-Exorista mimula

-Exorista rustica

-Exorista rustica

-Exorista rustica

-Exorista rustica

-Hyalurgus tomostethi

-Compsilura concinnata

-Triarthria setipennis

\section{Host record bibliography}

[1] Brauer, F. \& Bergenstamm, J. E. v. 1891: Die Zweiflügler des Kaiserlichen Museums zu Wien. V. Vorarbeiten zu einer Monographie der Muscaria schizometopa (exclusive Anthomyidae), Pars. II. - Denkschriften der Akademie der Wissenschaften in Wien, mathematisch-naturwissenschaftliche Klasse 58: 305-446.

[2] Brauer, F. \& Bergenstamm, J. E. v. 1894: Die Zweiflügler des Kaiserlichen Museums zu Wien. VII. Vorarbeiten zu einer Monographie der Muscaria schizometopa (exclusive Anthomyidae), Pars. IV. - Denkschriften der Akademie der Wissenschaften in Wien, mathematisch-naturwissenschaftliche Klasse, 61: 537-624.

[3] Cagáň, L., Turlings, T., Bokor, P. \& Dorn, S. 1999. Lydella thompsoni Herting (Dipt., Tachinidae), a parasitoid of the European corn borer, Ostrinia nubilalis Hbn. (Lep., Pyralidae) in Slovakia, Czech Republic and south-western Poland. - Journal of Applied Entomology 123: 577-583.

[4] Čapek, M. \& Čepelák, J. 1970: Zoznam parazitov dochovaných z hmyzích škodcov. Část' IV, Tachíny a mäsiarky (Diptera) [List of parasites brought up from insectean pests. Part IV, Tachinidae and Sarcophagidae (Diptera)]. - Pol'nohospodárstvo 16: 254 268. [In Slovak; Engl. \& Russ. summ.].

[5] Čapek, M. \& Čepelák, J. 1981: Zoznam parazitov dochovaných z hmyzích škodcov. VII. Tachíny a Mäsiarky [List of parasites bred from insect pests. Part VII. Tachinidae and Sarcophagidae]. - Pol'nohospodárstvo 27: 321-332. [In Slovak; Engl. \& Russ. summ.].

[6] Čepelák, J. 1952: II. Př́ispěvek k poznání Českých kuklic [IIe contribution à la connaissance des Tachinaires tchèques]. - Acta Societatis entomologicae Čechosloveniae 49: 169-180. [In Czech; French \& Russ. summ.].

[7] Čepelák, J. 1955a: Př́íspěvek k poznání slezských kuklic (Diptera-Larvevoridae) [Beitrag zur Kenntnis der schlesischen Raupenfliegen]. - Přrrodovědecký sbornik Ostravského kraje 16: 222-233. [In Czech; Russ. and Germ. summ.].

[8] Čepelák, J. 1955b: Př́íspěvek k poznání kuklic (Diptera-Larvevoridae) bourovce prsténčivého (Malacosoma neustrium L.) [Beitrag zur Kenntnis der Raupenfliegen des Ringelspinners (Malacosoma neustrium L.)]. — Zoolo- 
gické a entomologické listy [Folia zoologica et entomologica] 4: 167-174.

[9] Čepelák, J. 1963a: Eschenblattwespe Tomostethus nigritus F. als Wirt einer neuen Raupenfliege Hyalurgus tomostethi, n. sp. (Diptera, Larvaevoridae). — Biológia, Bratislava 18: 756-759.

[10] Čepelák, J. 1963b: Př́íspevok k poznaniu kuklíc (Diptera-Larvevoridae) mnišky zlatorítky (Euproctis phaeorrhoea Dun.) [Contribution to the knowledge of brown-tail moths (Euproctis phaeorrhoea Dun.) tachinids (Diptera Larvaevoridae)]. - Acta Universitatis Agriculturae (Nitra) 7: 125-136. [In Slovak, Engl. \& Russ. summ].

[11] Čepelák, J. 1963c: Príspevok k poznaniu kuklíc Slovenska (Larvaevoridae-Diptera) II [Contribution to the knowledge of the tachinids of Slovakia (Larvaevoridae-Diptera) II]. - Biologische Arbeiten 9/8, Entomologische Probleme 3: 57-85. [In Slovak, Engl. \& Russ. summ].

[12] Čepelák, J. 1997: Tachinidae: Part 1. — In: Vaňhara, J. \& Rozkošný, R. (eds), Faunistic records from the Czech and Slovak Republics: Diptera. Dipterologica bohemoslovaca Vol. 8. — Acta Facultatis scientiarum naturalium Universitatis Masarykianae Brunensis, Biologia 95: 235.

[13] Cziček, K. 1906: Beiträge zu einer Dipterenfauna Mährens. 1. Die Umgebung von Brünn. — Zeitschrift des mährischen Landesmuseums 6: 182-234.

[14] Finlayson, L. R. \& Finlayson, T. 1958a: Notes on parasites of Diprionidae in Europe and Japan and their establishment in Canada on Diprion hercyniae (Htg.) (Hymenoptera: Diprionidae). Canadian Entomologist 90 : 557-563.

[15] Finlayson, L. R. \& Finlayson, T. 1958b: Notes on parasitism of a spruce sawfly, Diprion polytomum (Htg.) (Hymenoptera: Diprionidae), in Czechoslovakia and Scandinavia. _ Canadian Entomologist 90: 584-589.

[16] Gold, J. 1893: Die Raupenfliegen. — Centralblatt für das gesamte Forstwesen 19: 300-305.

[17] Gold, J. 1895: Entomologische Studien. — Naturalien-Cabinet 13: 196-197; 14, 209-210; 15, 225-226; 16, 241242.

[18] Herting, B. 1960: Biologie der westpaläarktischen Raupenfliegen (Dipt., Tachinidae). — Monografien zur angewandten Entomologie Vol. 16. Parey, Hamburg, Berlin. 188 pp.

[19] Herting, B. 1961: Beiträge zur Kenntnis der europäischen Raupenfliegen (Dipt., Tachinidae), III-VI, — Stuttgarter Beiträge zur Naturkunde 65: 1-12.

[20] Hochmut, R. 1959: Př́íspěvek k poznání morphologie, bionomie a populační dynamiky obaleče hlohového ( $C a$ coecia crataegana $\mathrm{Hb}$.) [A contribution to the study of the morphology, bionomics and population dynamics of Cacoecia crataegana $\mathrm{Hb}$.]. — Práce výzkumných ústavů lesnických CSR 16: 23-58. [In Czech; Germ. \& Russ. summ.].

[21] Hochmut, R. 1964: Populační dynamika obaleče hlohového (Archips crataegana [Hb.]) v Dubinách ČSSR v letech 1957-61 [Population dynamics of Archips crataegana (Hb.) in the oak stands of Czechoslovakia in 19571961]. — Práce výzkumných ústavů lesnických ČSR 28: 35-80. [In Czech; English summ.].

[22] Holik, O. 1937: Die Biologie von Zygaena punctum O. — Entomologische Rundschau 54: 3940.

[23] International Organisation for Biological and Integrated Control of Noxious Animals and Plants, 1989: - Determination list of entomophagous insects Nr. 11. IOBC/WPRS Bulletin 12 (7), 63 pp.

[24] International Organisation for Biological and Integrated Control of Noxious Animals and Plants, 1993: - Determination list of entomophagous insects Nr. 12. IOBC/WPRS Bulletin 16 (3), 56 pp.

[25] International Organisation for Biological and Integrated Control of Noxious Animals and Plants, 2005: - Determination list of entomophagous insects Nr. 14. IOBC/WPRS Bulletin 28 (11), 71 pp.

[26] Jacentkovský, D. 1933a: Výskyt vzácných kuclic (Tachinidae) v ČSR [Occurrence of rare tachinids (Tachinidae) in Czechoslovak Republic]. — Sborník Vysoké školy zemědělské v Brně D, 20: 1-7. [In Czech].

[27] Jacentkovský, D. 1933b: Entomologické výzkumy v lesích Adamovských v letech [Entomological investigations in forests near Adamov in 1930-1932]. — Lesnická práce 12: 265-289. [In Czech].

[28] Jacentkovský, D., 1934. Kuklice (Tachinidae) "Masarykova lesa" [Tachinidae from Masaryk forest]. — Sborník vysoké Školy zemědělské v Brně D, 22, 1-38, 1 pl. [In Czech; French summ.].

[29] Jacentkovský, D. 1941a: K tachinologickému výzkumu školního statku vysoké školy zemědělské v Brně [Zur tachinologischen Durchforschung des Schulforstgutes der landwirtschaftlichen Hochschule in Brünn]. — Sborník entomologického oddělení Zemského Musea v Praze 19: 76-80. [In Czech; Germ. summ.].

[30] Jacentkovský, D. 1941b: Kuklice (Tachinoidea, Diptera) Moravy a Slezska [Die Raupenfliegen (Tachinoidea) Mährens und Schlesiens]. - Acta Societatis scientiarum naturalium Moravicae 13 (4): 1-64. [In Czech; Germ. summ.].

[31] Jacentkovský, D. 1942a: Tachinologická miscelanea [Tachinologische Miscelanea]. — Sborník entomologického oddělení Zemského Musea v Praze 20: 127-131. [In Czech; Germ. summ.].

[32] Jacentkovský, D. 1942b: K tachinologickému výzkumu školního statku vysoké školy zemědělské v Brně (Pokračování) [Zur tachinologischen Durchforschung des Schulforstgutes der landwirtschaftlichen Hochschule in Brünn (Fortsetzung)]. — Sborník entomologického oddělení Zemského Musea v Praze 20: 172-187. [In Czech; Germ. summ.].

[33] Kaděra, M. 1969: Oberea pupillata (Gyll.) (Col., Cerambycidae) - nový hostitel Billaea irrorata Meig. (Dip., Dexinae) [Oberea pupillata (Gyll.), (Col., Cerambycidae) ein neuer Wirt von Billaea irrorata Meig. (Dip., Dexi- 
nae)]. - Zprávy Československé společnosti entomologické pri Československé akademii věd 5: 50. [In Czech; Germ. summ.].

[34] Kalandra, A. 1961: Verlauf der Massenvermehrung der gemeinen Gespinstblattwespe Cephaleia abietis L. im Erzgebirge in den Jahren 1950-1960. — Communicationes Instituti forestalis Čechosloveniae 2: 173-176.

[35] Kirchner, L. 1861: Zur Biologie der Sphinx pinastri. — Lotos 11: 103-104.

[36] Kletečka, Z. 1996: Nálezy Dipter (Tipulidae, Tachinidae, Xylophagidae) zajímavé pro faunu Čech [Remarkable records of Diptera (Tipulidae, Tachinidae, Xylophagidae) from Bohemia]. - Sborník Jihočeského muzea v Českých Budějovicích, Př́rodní vědy [Acta Musei Bohemia meridionalis in České Budějovice, Scientiae naturales] 36: 65-67. [In Czech; Engl. summ.].

[37] Kolubajiv, S. 1934: Zkušenosti s pěstováním parasitických druhů hmyzu z jejich hostitelů, získané ve Státním výzkumném ústavě pro ochranu lesů v Praze v roce 1929-1933 [Die Ergebnisse der Züchtung von parasitischen Insektenarten aus ihren Wirten in der staatlichen Versuchsanstalt in Prag in den J.J. 1929-1933]. — Časopis České společnosti entomologické 31: 59 68, 113-120, 155-163. [In Czech; Germ. summ.].

[38] Kolubajiv, S. 1938: Příspěvek k biologii pilatky ryšavé [Beitrag zur Biologie der rotgelben Kiefernbuschhornblattwespe (Diprion sertifer Geoffr. Lophyrus rufus Kl.). — Lesnická práce 17: 325-348.

[39] Kolubajiv, S. 1962: Výsledky chovu entomofágů (cizopasníků a dravců) hmyzích škůdců — hlavně lesních získaných v letech 1934-1958 [Die Ergebnisse der Zuchten von Entomophagen (der Parasiten und Räuber) der schädlichen Insekten (vorwiegend der Forstschädlinge) in der Zeitperiode von 1934 bis 1958. — Rozpravy Československé akademie věd, řada matematických a prírodních věd 72 (6): 1-73. [In Czech; Russ. and Germ. summ.].

[40] Komárek, J. 1933: Wichtige Neubeobachtungen aus der Biologie der Nonne. — Anzeiger für Schädlingskunde, 9 (6-7), 77-82, 93-96.

[41] Komárek, J. \& Kolubajiv S. 1941: Einige Beobachtungen über den Kiefernspinner. — Centralblatt für das gesamte Forstwesen 67: 245-251.

[42] Křístek, J. \& Petruška, F. jr. 1982. Gradation der Kiefernbuschhornblattwespe Diprion pini (L.) (Hymenoptera, Diprionidae) im Forstbetrieb Strážnice (Südostmähren). - Acta Universitatis agriculturae (Brno), Series C (Facultas silviculturae) 51: 83-106.

[43] Lemarie, J. 1959: Př́spěvek k póznaní cizopasníků makadlovky borové Exoteleia (Heringia) dodecella L. Část II. Čeledi Ichneumonidae, Braconidae, Larvaevoridae [Beitrag zur Kenntnis der Parasiten der Kieferknospentriebmotte Exoteleia (Heringia) dodecella L. Teil 2. Ichneumonidae, Braconidae und Larvaevoridae]. — Zoologické listy [Folia zoologica] 8: 309-314. [In Czech; Germ. summ.].

[44] Loos, K. 1908: Beobachtungen über einen bedeutungsvollen Fliegenschmarotzer an dem Nonneninsekte. - Centralblatt für das gesamte Forstwesen 34: 4-9.

[45] Loos, K. 1909: Parasetigena segregata Rdi. und einige andere Schädiger des Nonneninsektes. — Centralblatt für das gesamte Forstwesen 35: 427-431.

[46] Loos, K. 1911: Weitere Beobachtungen an Parasetigena segregata. — Forst- und Jagdzeitung - Fachschrift des deutschen Forstvereines für Böhmen 11: 435-438.

[47] Loos, K. 1916: Einige Beobachtungen, Versuche und Untersuchungen über die Lebensweise der Tachine Parasetigena segregata Rdi auf dem Libocher Herrschaftsgebiet. — Vereinsschrift für Forst-, Jagd- und Naturkunde 1916: 1-27.

[48] Martinek, V. 1972: Die Übervermehrung der Roten Kiefernbuschhornblattwespe (Neodiprion sertifer Geoffr.) und die Bedeutung ihrer Parasiten in Knieholzbeständen. - Rozpravy Československé akademie věd, řada matematických a př́rodních věd $82(5): 1-115$.

[49] Martinek, V. 1980: Zum Problem der Übervermehrung der gemeinen Fichtengespinstblatttwespe (Cephalcia abietis L.) (Hym., Pamphiliidae) in Böhmen. — Rozpravy Československé akademie věd, řada matematických a př́rodních věd 90 (4): 1-165.

[50] Martinek, V. 1984 Parasitoids of the European pine shoot moth Rhyacionia buoliana (Schiff.) in Bohemia and Moravia. - Studie Československé akademie věd 14: 1-113.

[51] Martinek, V. 1985: Egg and larval parasites of the European sawfly Neodiprion sertifer (Geoff.) in Bohemia. Studie Československé akademie věd 26: 1-123.

[52] Martinek, V. 1987: Cizopasníci ploskohřbetek rodu Cephalcia Pz. (Hym., Pamphiliidae) v ČSR. I. Voltinismus kuklice Myxexoristops abietis Hert. (Dipt., Tachinidae) [The parasites of web-spinning sawflies of the Cephalcia Pz. genus (Hym., Pamphiliidae) in the CSR. Part I. Voltinism of the fly Myxexoristops abietis Hert. (Dipt., Tachinidae)]. - Lesnictví 33: 583-606. [In Czech; Russ. \& Engl. summ.].

[53] Martinek, V. 1990: Cizopasníci ploskohřbetek rodu Cephalcia Pz. (Hym., Pamphiliidae) v ČR. V. Zastoupení larválních cizopasníků ve spektru přirozených nepřátel [Parasites of web-spinning sawflies of the Cephalcia Pz. genus (Hym., Pamphilidae) in the ČR. Part V. Representation of larval parasites in the spectrum of natural enemies]. — Lesnictví 36: 201-240. [In Czech; Germ., Engl. \& Russ. summ.].

[54] Martinek, V. 1991: Přemnožení ploskohřbetky severské Cephalcia arvensis Panz. (Hym., Pamphilidae) ve východních Čechách [An outbreak of the web-spinning sawfly Cephalcia arvensis Panz. (Hym., Pamphilidae) in 
eastern Bohemia]. - Lesnictví 37: 543-570. [In Czech; Engl. summ.].

[55] Martinek, V. 1992: Nový škůdce smrku, ploskohřbetka černá (Cephalcia falleni Dalm.) (Hym., Pamphilidae), v Orlických horách [The sawfly (Cephalcia falleni Dalm.) (Hym., Pamphilidae) as a new pest on spruce in the Orlické Mts.]. — Lesnictví-Forestry 38: 205-220. [In Czech; Engl. summ.].

[56] Martinek, V. 1994: Pilatka horská [Pikonema montana (Zadd.), Hymenoptera, Tenthredinidae], významný škůdce smrku v pahorkatině severovýchodních Čech [The sawfly Pikonema montana (Zadd.) (Hym., Tenthredinidae), an important pest on spruce in the hills of North-East Bohemia]. — Lesnictví-Forestry 40: 139-149. [In Czech; Engl. summ.].

[57] Martinek, V. \& Kudler, J. 1964: Chemická ochrana kleče v Krušných horách před žírem hřebenule Neodiprion sertifer (Geoffr.) a vliv na parasitofaunu [Chemical protection of the mountain pine in the Krušné hory Mountains against the feeding of Neodiprion sertifer (Geoffr.) and influence on the parasites]. - Práce výzkumných ústavů lesnických ČSSR 29: 1-260. [In Czech; Russ. and Engl. summ.].

[58] Mik, J. \& Wachtl, F. A. 1895: Commentar zu den Arbeiten von Hartig und Ratzeburg über Raupenfliegen (Tachiniden). Auf Grund einer Revision der Hartig'schen Tachiniden-Sammlung. - Wiener entomologische Zeitung 14: $213-250$.

[59] Morris, K. R., Cameron, E. \& Jepson, W. F. 1937: The insect parasites of the spruce sawfly (Diprion polytomum, Htg.) in Europe. - Bulletin of Entomological Research 28: 341-393.

[60] Mrkva, R. 1965: Př́spěvek k morfologii, bionomii a poznání parazitů pilatky jasanové (Tomostethus nigritus [Fabr.]) [Beitrag zur Kenntnis der Morphologie, Bionomie und der Parasiten der schwarzen Eschenblattwespe (Tomostethus nigritus [Fabr.]). — Práce výzkumných ústavi̊ lesnických ČSSR 30: 33-64.

[61] Mrkva, R. 1968: Polulační dynamika píd’alky podzimní (Operophthera brumata L.) během její epifytotie v letech 1962-1965 [The population dynamics of the Small Winter Moth (Operophthera brumata L.) during its epiphytic stage in 1962-1965]. — Lesnický časopis 14: 317-338. [In Czech; Russ., Engl. \& Germ. summ.].

[62] Mückstein, P., Tschorsnig, H.-P. \& Vaňhara, J. 2004: Some new host records of West Palaearctic Tachinidae (Diptera). - In: Bitušík, P. (ed.), Dipterologica bohemoslovaca. Vol. 12. - Acta Facultatis Ecologiae, Zvolen 12: $111-113$.

[63] Mückstein, P., Tschorsnig, H.-P., Vaňhara, J. \& Michalková, V. 2007: New host and country records for European Tachinidae (Diptera). - Entomologica Fennica 18: 179-189.

[64] Organisation Internationale de Lutte Biologique contre les animaux et les plantes nuisibles, 1957: Liste d'identification No 2. - Entomophaga 2: 313-332.

[65] Pfeffer, A. 1930a Nový nebezpečný škůdce smrku zavíječ modř́nový — Enarmonia (Epinotia, Steganoptycha) diniana Z. (pinicolana Z.) [Der graue Lärchentriebwickler (Enarmonia diniana Z.) als gefährlicher Fichtenschädling]. - Ochrana rostlin 10: 81-95. [In Czech; Germ. summ.].

[66] Pfeffer, A. 1930b: Zavíječ modř́nový Enarmonia (Epinotia, Steganoptycha) diniana Gn. (pinicolana Z.) [Der Lärchentriebwickler - Enarmonia diniana Gn.]. —Lesnická práce 9: 1-24. [In Czech; Germ. summ.].

[67] Pschorn-Walcher, H. 1965: The ecology of Neodiprion sertifer (Geoffr.) (Hym.: Diprionidae) and a review of its parasite complex in Europe. - Technical Bulletin of the Commonwealth Institute of Biological Control 5: 3397.

[68] Rambousek, F. 1928: Škůdcové a ochránci řepní. I. [Pests and bioregulators of the beet I.]. Zoologická část řepařské fytopatologie. - Publikace Ministerstva zemědělství RČS. 74: 1-415. [In Czech].

[69] Rambousek, F. 1929: Die Rübenschädlinge im Jahre 1927 und 1928. — Zeitschrift für die Zuckerindustrie der Čechoslovakischen Republik 54: 105-114.

[70] Rambousek F. \& Straňák, F. 1919: Př́ispěvek k studiu můry osenní (Agrotis segetum). [Contribution to the study of Agrotis segetum]. — Zemědělský archiv 10: 24-34.

[71] Riedel, M. P. 1930: Mitteilungen der Sammelstelle für Schmarotzerbestimmungen des V.D.E.V., Tachinidae I. - Entomologischer Anzeiger 10:262-266.

[72] Riedel, M. P. 1931: Mitteilungen der Sammelstelle für Schmarotzerbestimmungen des V.D.E.V., Tachinidae II. - Entomologischer Anzeiger 11:397-400.

[73] Růžička, J. 1923: České tachiny (chotovinské a jiné) [Czech tachinids (from Chotoviny and others)]. — Lesnická práce 2: $571-572$.

[74] Růžička, J. 1932: Nová pozorování mnišky a tachin z r. 1930 a 1931 [Neue Beobachtungen über das Erscheinen der Nonne und der Tachinen in den Jahren 1930 und 1931]. — Lesnická práce 11: 70-91, 124-145. [In Czech; Germ. summ.].

[75] Růžička, J. 1933: Další pozorování mnišky a tachin z r. 1932 [Weitere Beobachtungen der Nonne und der Tachinen im J. 1932]. —- Lesnická práce 12: 289-321. [In Czech; Germ. summ.].

[76] Růžička, J. 1934: Dokončení pozorování mnišky a tachin z r. 1933 [Schlussbeobachtungen der Nonne und der Tachinen im J. 1933]. — Lesnická práce 13: 149-170. [In Czech; Germ. summ.].

[77] Řezáč, M. 1950: Kuklice rodu Viviana, ničitelé hrbáče osenního [Tachinidae of gen. Viviania parasiting on Zabrus tenebrioides F.]. — Entomologické listy [Folia entomologica] 13: 46 47. [In Czech; English summ.].

[78] Řezáč, M. 1959: Několik poznámek k problému makadlovky kmínové Depressaria nervosa Hw, nejvážnějšího 
škůdce okoličnatých rostlin, zvláště kmínu, u nás [Zum Problem der Kümmelmotte, Depressaria nervosa Hw. (Lep.), eines Schädlings der Doldenpflanze — insbesondere des Kümmels — in der Tschechoslowakei. — Zoologické listy [Folia zoologica] 8: 1-19. [In Germ., partly in Czech].

[79] Samšiňáková, A. \& Samšiňák, K. 1957: Nepřátelé a nemoci zádumčivce olšového (Agelastica alni L.) [Feinde und Krankheiten der Agelastica alni L.]. —Lesnický časopis 3: 317-322. [In Czech].

[80] Schimitschek, E. 1935: Forstschädlingsauftreten in Österreich 1927 bis 1933. — Centralblatt für das gesamte Forstwesen 61 (7-8): 208-221.

[81] Schimitschek, E. 1943: Untersuchungen über Parasitenreihen. - Mitteilungen der Akademie der deutschen Forstwissenschaft 3: 272-305.

[82] Schimitschek, E. 1964: Liste der 1934-1936 und 1940-1953 gezogenen Parasiten und ihrer Wirte. — Zeitschrift für angewandte Entomologie 53: 320-341.

[83] Šámal, J. 1940: Př́spěvek k oekologii obaleče Acalla ferrugana Tr. [Contribution to the ecology of Acalla ferrugana Tr.]. - Acta Societatis entomologicae Bohemiae 8: 131-148. [In Czech; French summ.].

[84] Šedivý, J. 1959: Einfluß der chemischen Bekämpfung auf die Entomophagen in der Zeit der Graseulen-Kalamität (Charaeas graminis L.). - In: Transactions of the 1st international Conference on Insect Pathology and Biological Control, Praha 1958: 503-507.

[85] Šedivý, J. 1969: Die Ursachen von Massenvermehrungen der Graseule (Cerapteryx graminis Linné) im Erzgebirge. - Bericht über die 10. Wanderversammlung deutscher Entomologen: 355-363.

[86] Tölg, F. 1913: Biologie und Morphologie einiger in Nonnenraupen schmarotzender Fliegenlarven. - Centralblatt für Bakteriologie und Parasitenkunde, Abteilung 2, 37: 392-412.

[87] Tschorsnig, H.-P. \& Herting, B. 2005: Die Raupenfliegen-Sammlung Friedrich A. Wachtl (Diptera: Tachinidae). — Veröffentlichungen des Tiroler Landesmuseums Ferdinandeum 84 (2004): 181-236.

[88] Urban, J. 1993: Výskyt a vývoj pilatky Nematus (Pteronidea) melanaspis Htg. (Tenthredinidae, Hymenoptera) na vrbách pěstovaných na plantážích na Moravě [The occurrence and development of the sawfly Nematus (Pteronidea) melanaspis Htg. (Tenthredinidae, Hymenoptera) in willows grown in plantations in Moravia]. - LesnictvíForestry 39: 129-137. [In Czech; Engl. summ.].

[89] Urban, J. 1995: On the occurrence, bionomics and harmfulness of Altica quercetorum quercetorum Foud (Coleoptera, Alticidae). - Lesnictví-Forestry 41: 497-510.

[90] Urban, J. 1998a: Př́spěvek k poznání mandelinky Gonioctena (=Phytodecta) quinquepunctata F. (Chrysomelidae, Coleoptera) [A contribution to the knowledge of a chrysomelid beetle Gonioctena (=Phytodecta) quinquepunctata F. (Chrysomelidae, Coleoptera)]. - Acta Universitatis agriculturae et silviculturae Mendelianae Brunensis 46 (1): 7-23. [In Czech; Engl. summ.].

[91] Urban, J. 1998b: Insect parasitoids of the chrysomelid Chrysomela vigintipunctata. — Acta Universitatis agriculturae et silviculturae Mendelianae Brunensis 46 (4): 13-39.

[92] Urban, J. 1999: Výsledky studia biologie a škodlivosti bázlivce olšového (Agelastica alni L.) (Chrysomelidae, Coleoptera) [Results of the study of biology and harmfulness of alder leaf beetle (Agelastica alni L.) (Chrysomelidae, Coleoptera)]. - Acta Universitatis agriculturae et silviculturae Mendelianae Brunensis 47 (5): 47-71. [In Czech; Engl. summ.].

[93] Urban, J. 2000: Výskyt, bionomie a škodlivost mandelinky olšové (Linaeidea aenea L.) [Incidence, bionomics and harmfulness of the alder chrysomelid beetle (Linaeidea aenea L.)]. - Lesnictví-Forestry 46: 468-484. [In Czech; Engl. summ.].

[94] Urban, J. 2005: Contribution to the knowledge of development and harmfulness of imported willow leaf beetle (Plagiodera versicolora) (Coleoptera, Chrysomelidae). - Journal of Forest Science 51: 481-507.

[95] Urban, J. 2006: Occurrence, bionomics and harmfulness of Chrysomela populi L. (Coleoptera, Chrysomelidae). - Journal of Forest Science 52: 255-284.

[96] Urban, J. 2007: Occurrence, biology and harmfulness of Galerucella lineola (F.) (Coleoptera, Chrysomelidae). Part 1. Last year's (parent) beetles. Part 2. Larvae and this year's beetles. — Journal of Forest Science 53: 364 $380,424-444$.

[97] Urban, J. \& Šedivý, J. 1997: Faktory regulující přemnožení štětconoše ořechového (Calliteara pudibunda L.) (Lepidoptera, Lymantriidae) [Factors regulating the mass outbreak of the pale tussock moth (Calliteara pudibunda L.) (Lepidoptera, Lymantriidae)]. — Lesnictví-Forestry 43: 67-78. [In Czech; Engl. summ.].

[98] Vaňhara, J., Tschorsnig, H.-P. \& Barták, M. 2004: New records of Tachinidae (Diptera) from the Czech Republic and Slovakia, with a revised check-list. — Studia dipterologica 10 (2003): 679-701.

[99] Vimmer, A. 1906: Doplňky ke Kowarzovu seznamu českých dipter. - Acta Societatis entomologicae bohemiae 3: 88-89. [In Czech].

[100] Vimmer, A. 1907: Mouchy, které cizopasí v larvách a kuklách některých českých motýlů [Die Fliegen als Parasiten einiger böhmischer Schmetterlinge]. — Acta Societatis entomologicae bohemiae 4: 1-4. [In Czech; Germ. summ.].

[101] Vimmer, A. 1909: Mouchy, které cizopasí v larvách a kuklách některých českých motýlů. (2. př́spěvek) [Die Fliegen als Parasiten einiger böhmischer Schmetterlinge. (2. Beitrag)]. - Acta Societatis entomologicae bohemi- 
ae 6: 65-66. [In Czech; Germ. summ.].

[102] Vimmer, A. 1911: Příspěvky k poznávání kukel hmyzu dvojkř́́dlého. Diptera cyclorrhapha. Část prvá [Beiträge zur Kenntnis der cyclorrhaphen Dipterenpuppen. I. Teil. Schizometopa). - Acta Societatis entomologicae bohemiae 8: 34 43. [In Czech; Germ. summ.].

[103] Vimmer, A. 1913: Seznam českého hmyzu dvoukřídlého [Catalogus dipterorum]. — Entomologické přríručky 8: 1-99. [In Czech].

[104] Vimmer, A. 1925: Larvy a kukly dvojkřŕdlého hmyzu středoevropského [Larvae and pupae of central European Diptera]. - Česká grafická unie a.s., Praha. 348 pp. +59 pls. [In Czech].

[105] Vimmer, A. 1928: Muší parasiti českých motýlů. III. [Die Fliegenparasiten der böhmischen Falter. III.]. — Acta Societatis entomologicae Čechosloveniae 25: 48-52. [In Czech; Germ. summ.].

[106] Vimmer, A. 1929: Další parasiti muší českých motýlů (IV.) [Further parasites of Bohemian Lepidoptera]. — Acta Societatis entomologicae Čechosloveniae 26: 46 47. [In Czech].

[107] Vimmer, A. 1930: Muší parasiti českých motýlů. V. část. [Fly parasites of Bohemian butterflies. Part V.]. — Acta Societatis entomologicae Čechosloveniae 27: 54. [In Czech].

[108] Vimmer, A. 1932: Druhy Tachin ČSR ze starého, velkého rodu Exorista Mg. [Die Tachinenarten d. ČSR der alten grossen Exorista-Gattung]. - Acta Societatis entomologicae Čechosloveniae 29: 126-137. [In Czech].

[109] Vimmer, A. 1934: Nový parasit motýlů [New parasite of butterflies]. - Acta Societatis entomologicae Čechosloveniae 31: 189. [In Czech].

[110] Vimmer, A. 1935: Opět nový hostitel Tachin. [Again a new parasite of butterflies]. - Acta Societatis entomologicae Čechosloveniae 32: 46. [In Czech].

[111] Vimmer, A. 1938a: Parasiti českých motýlů. VII. [Parasites of Bohemian butterflies. VII.]. - Acta Societatis entomologicae Čechosloveniae 35: 62. [In Czech].

[112] Vimmer, A. 1938b: Noví cizopasníci českých motýlů. [New parasites of Bohemian butterflies]. — Acta Societatis entomologicae Čechosloveniae 35: 125-126. [In Czech].

[113] Wachtl, F. A. 1882: Beiträge zur Kenntnis der Biologie, Systematik und Synonymie der Insekten. — Wiener entomologische Zeitung 1:275-279.

[114] Wachtl, F. A. 1886: Einige Resultate meiner Zuchten. - Wiener entomologische Zeitung 5: 307.

[115] Weiser, J. 1960: Nemoci ponrav chroustů v ČSR [Infections of Melolontha larvae in Czechoslovakia]. — Acta Societatis zoologicae Bohemoslovenicae 24: 71-74. [In Czech; Engl. summ.].

[116] Zuska, J. 1963: The puparia of the European species of the family Larvaevoridae (Diptera) I (Subfamily Salmaciinae, part 1). - Acta entomologica Musei nationalis Pragae 35: 333-372.

\section{Discussion}

The present catalogue lists 195 hosts for 149 tachinid species, but it must be taken into consideration that some of the older tachinid identifications or host associations are wrong or most probably wrong. The bibliography for the host records consists of 116 papers, which were based on the work of 55 researchers.

The majority of the Czech host records concerns the subfamily Exoristinae (103 tachinid species, 579 records of 332 host-parasitoid couples, making $82 \%$ of all Czech host records) and to a lesser extent Tachininae (33 tachinid species, 91 records of 54 host-parasitoid couples, making $13 \%$ of all Czech host records) and Dexinae (13 tachinid species, 23 records of 34 host-parasitoid couples, making 5\% of all Czech host records). The average for the Palaearctic region is not much different $(74 \%$ of the host records belong to Exoristinae, $13 \%$ to Tachininae, $7 \%$ to Dexiinae, and $6 \%$ to Phasiinae). There are no rearing re- cords of Phasiinae for the Czech Republic because their hosts (Hemiptera) do not play a special role as pests in this country. Members of Orthoptera and Diptera are neither usually important as pests in the Czech Republic. Accordingly, there is not a single record for these host orders. Most hosts in the Czech Republic have the unspecialized parasitoids Exorista larvarum, Blondelia nigripes, Compsilura concinnata and Phryxe vulgaris, but this is not different from what is is known from other European countries.

Investigations on tachinid-host relations in the Czech Republic were primarily focused on pests in forestry, so it is not surprising that records for such typical hosts as Lymantria monacha, Dendrolimus pini, Euproctis chrysorrhoea, $\mathrm{Ma}$ lacosoma neustria and Tortrix viridana rank first. But also some common host species which are reared by practically every lepidopterologist (e.g. Aglais urticae, Vanessa io, Saturnia pavonia etc.) are represented by many tachinid records. Larvae of "unimpressive" hosts or of those which feed 
hidden on roots or in stems, which live in or near the soil, or which show a nocturnal behaviour are, however, underrepresented - as usual. Future search on interesting tachinid hosts should concentrate on such animals.

Acknowledgements. For financial support we are indebted to the Ministry of Education/Masaryk University for the grants No. MSM 0021622416 and the Grant Agency CR $524 / 05 /$ H536. We are grateful to I. Adamová (Brno, CZ) for her help with finding tachinology literature. English was kindly revised by P. J. Chandler (Melksham, Wiltshire, GB).

\section{References}

Andersen, S. 1988: Revision of European species of $P h y$ tomyptera Rondani (Diptera, Tachinidae). — Entomologica Scandinavica 19: 43-80.

Andersen, S. 1996: The Siphonini (Diptera: Tachinidae) of Europe. - Fauna Entomologica Scandinavica 33: 1148.

Baer, W. 1921: Die Tachinen als Schmarotzer der schädlichen Insekten. Ihre Lebensweise, wirtschaftliche Bedeutung und systematische Kennzeichnung. - Zeitschrift für angewandte Entomologie 7: 97-163, 349 423.

Herting, B. \& Dely-Draskovits, A. 1993: Family Tachinidae. - In: Soós, A. \& Papp, L., Catalogue of Palaearctic Diptera. Vol. 13: 118-624. Hungarian Natural History Museum, Budapest. 624 pp.

Jelínek, J. (ed.) 1993: Seznam československých brouků [Check-list of Czechoslovak Insects IV (Coleoptera)]. - Folia Heyrovskyana, Suppl. 1: 1-172.

Koleška, Z. 1993: Seznam biografií československých entomologů (entomologové nežijící), 14. pokračování. Heslo: Stein Richard, rytír, Med. Dr. [List of Czechoslovak entomologist biographies (entomologists no-alive), 14th Continuation. Item: Stein Richard, Knight, Med. Dr.]. — Klapalekiana, Suppl. 29: 544.

Kolubajiv, S. 1937: Poznámky k biologii mnišky a jejích hlavních hmyzích parasitů [Notes on the biology of the nun moth and its main parasite insects]. - Lesnická práce 16: 169-199. [In Czech; Germ, and Engl. summ.].

Kolubajiv, S. \& Pfeffer, A. 1931: Parasitický hmyz na mnišce a pomniškoví kůrovci. I. Tachiny. Seznam parasitických tachin: 197-198; Stručný popis nejdůležitějších parasitů mnišky: 200-201; Klíč k určování podčeledí a rodů tachin parasitujících na mnišce: 202 207. [Insect parasitoids on L. monacha and "after monacha's" bark beetles. I. Tachinids. List of tachinid pa- rasitoids: 197-198; Short description of the most important parasitoids of L. monacha: 200-201; Key to the identification of tachinid subfamilies and genera parasitizing on L. monacha: 202-207]. — In: Komárek, J. (ed.), Mnišková kalamita v létech 1917-1927. [Disaster of L. monacha during 1917-1927]. Sborník výzkumných ústavů zemědělských ČSR. Vol. 78. Praha. [In Czech; Germ. summ.].

Kudler, J. 1954: Mniška a boj proti ní. [Lymantria monacha and struggle against it] — Lesnická knihovna, M. ř. Vol. 40. Státní zemědělské nakladatelství, Praha. 52 pp. [In Czech].

Lašti̊vka, Z. \& Liška, J. 2005: Seznam motýlů České republiky [Checklist of Lepidoptera of the Czech Republic] (Insecta: Lepidoptera), 40. [www document]. URL http:/www.lepidoptera.wz.cz/Lepidoptera.pdf (Accessed Aug. 20, 2007).

Preyssler, J. D., Lindacker, J. C. \& Hofer, J. K. 1793: Beobachtungen über Gegenstände der Natur, auf einer Reise durch den Böhmerwald im Sommer 1791. Sammlung physikalischer Aufsätze, Dresden 3: 135 378.

Shima, H. 1996: A systematic study of the tribe Winthemiini from Japan (Diptera: Tachinidae). — Beiträge zur Entomologie, Berlin 46: 169-235.

Tschorsnig, H.-P. \& Herting, B. 1994: Die Raupenfliegen (Diptera: Tachinidae) Mitteleuropas: Bestimmungstabellen und Angaben zur Verbreitung und Ökologie der einzelnen Arten. - Stuttgarter Beiträge zur Naturkunde, Serie A (Biologie) 506: 1-170.

Tschorsnig, H.-P. \& Richter, V. 1998: Tachinidae. - In: Papp, L. \& Darvas, B. (eds) Contributions to a Manual of Palaearctic Diptera (with special reference to flies of economic importance). Higher Brachycera. Vol. 3 : 691-827. Science Herald, Budapest. 880 pp.

Tschorsnig, H.-P., Vaňhara, J., Barták, M. \& Kubík, Š. 2005: Tachinidae. - In: Barták, M. \& Kubík, Š . (eds), Diptera of Podyjí National Park and its environs: 398414. Czech University of Agriculture, Prague. 432 pp. (incl. CD).

Vaňhara, J. \& Tschorsnig, H.-P. 2006: Tachinidae Robineau-Desvoidy 1830. — In: Jedlička, L., Kúdela, M. \& Stloukalová, V. (eds): Checklist of Diptera of the Czech Republic and Slovakia. Electronic version 1, CD ROM edition, Comenius University, Bratislava. Available also online at URL http://zoology. fns.uniba. sk/diptera/Tachinidae.htm (Accessed Dec. 2007).

Ziegler, J. 2000: Tachinidae. — In: Ziegler, J. \& Menzel, F. (eds), Die historische Dipteren-Sammlung Carl Friedrich Ketel. Revision einer zwischen 1884 und 1903 angelegten Sammlung von Zweiflüglern (Diptera) aus Mecklenburg-Vorpommern: 201-229. Nova Supplementa Entomologica Vol. 14. Deutsches Entomologisches Institut, Berlin. 266 pp. 